\title{
Role of interfacial transition zone in phase field modeling of fracture in layered heterogeneous structures
}

\author{
Thanh-Tung Nguyen ${ }^{\mathrm{a}}$, Danièle Waldmann ${ }^{\mathrm{a}}$, Tinh Quoc Bui ${ }^{\mathrm{b}, \mathrm{c}, *}$ \\ ${ }^{a}$ Laboratory of Solid Structures, University of Luxembourg, \\ 6, Rue Richard Coudenhove-Kalergi, L-1359, Luxembourg \\ ${ }^{b}$ Institute for Research and Development, Duy Tan University, Da Nang City, Viet Nam. \\ ${ }^{c}$ Department of Civil and Environmental Engineering, Tokyo Institute of Technology, \\ 2-12-1-W8-22, Ookayama, Meguro-ku, Tokyo 152-8552, Japan.
}

\section{A R T I C L E IN F O}

Article history:

Keywords: fracture, layered structure, cohesive zone model, crack propagation, phase field model, finite element analysis.

\begin{abstract}
A B S T R A C T
Mechanical behavior of layered materials and structures greatly depends on the mechanical behavior of interfaces. In the past decades, the failure in such layered media has been studied by many researchers due to their critical role in the mechanics and physics of solids. This study aims at investigating crackinterface interaction in two-dimensional (2-D) and three-dimensional (3-D) layered media by a phase field model. Our objectives are fourfold: (a) to better understand fracture behavior in layered heterogeneous systems under quasi-static load; (b) to introduce a new methodology for better describing interfaces by a regularized interfacial transition zone in the context of variational phase field approach, exploring its important role; (c) to show the accuracy, performance and applicability of the present model in modeling material failure at the interfaces in both 2-D and 3-D bodies; and (d) to quantitatively validate computed crack path with respect to experimental data. Phase field models with both perfectly and cohesive bonded interfaces are thus derived. A regularized interfacial transition zone is introduced to capture characteristics of material mismatch at the interfaces. Numerical examples for 2-D and 3-D layered systems with experimental validation provide fundamentals of fracture behavior in layered structures. The obtained results shed light on the behavior of crack paths, which are drastically affected by the elastic modulus mismatch between two layers and interface types, and reveal the important role of the proposed interfacial transition zone in phase field modeling of crack-interface interactions.
\end{abstract}

(c) 2019 Elsevier Inc. All rights reserved. 


\section{Introduction}

\subsection{Background}

Recent advances in engineering and structural materials have enabled the developments of modern and advanced products that could improve the quality of our daily lives. However, they often require the usage of multi-components and structures which are fabricated by joining different types of materials, forming layered systems. In particular, layered structures of heterogeneous materials have thus received considerable attention, and are increasingly being used in many engineering applications ([58]). Although layered systems have great potential in applications, their mechanical behavior however heavily depends on the mechanical properties and performance of the interfaces. It is well-known that in layered systems the properties vary from layer to layer, leading to property discontinuity and therefore jumps across the interfaces. Due to the low fracture resistance, failure and defects, which are major issues encountered in engineering practice, can occur under severe loading conditions. The presence of property jumps, geometric discontinuity, or material mismatch at layered interfaces induces more complexities in the fracture modeling of the media. In fact, the change of elastic properties and fracture characteristics from layer to layer, type of loading experienced, crack orientation, etc. are certainly important factors that must be analyzed thoroughly.

The mechanical properties of layered structures generally could be tuned by manipulating the mechanical properties of interfaces. The knowledge of failure behavior at the interfaces in layered media thus is of great importance to the design of engineering applications, and it holds one major research subject to the scientific community. In the past decades, research efforts have been largely shifted towards the studies of this subject in both theoretical and computational models, for instance, see $([7,6,28,32])$ and references therein.

Fracture of crack-interface interactions in layered media has been analyzed using different numerical approaches. Linear Elastic Fracture Mechanics (LEFM) is the popular technique, where the fracture phenomena are often investigated through stress intensity factors (SIFs), toughness or asymptotic solutions. However, those aspects heavily depend on the material mismatch and interfacial behavior, e.g., see ([28, 7]. Previous experimental observations show that the interfacial region is often the origin and main source of failure ([1]), but an efficient numerical model possibly reproducing these phenomena is still missing. Fracture mechanics based models have demonstrated great potential in modeling crack growth in layered materials. However, this kind of approaches is relatively restricted and influenced not only by materials but also by geometry effects. Therefore, it becomes quite difficult in modeling complex layered structure, especially arbitrary interfaces and 3-D cases.

Cohesive zone models (CZM) is another popular technique to model the interfaces in layered systems ([53, 30, 62]). To avoid the singularity of stress at crack-tip, [23] and [8] introduced a cohesive zone where cohesive laws are used. Imperfect interface in layered media can be well modeled by the CZM. However, this type of structures presents different materials with different failure mechanisms, so that a complex model is needed to describe its fracture [71]. Another significant drawback of CZM is that the cohesive surfaces can only lie along element edges, which results in mesh-dependent crack paths.

The eXtended Finite Element Method - XFEM ([43]) is also used to study fracture in bimaterials interface and layered structures, e.g., see $([63,39,10,72])$. The advantage of XFEM in modeling crack propagation is that the finite element mesh does not need to be remeshing to track crack paths. The evolution of crack is independent of mesh, arbitrarily through elements, rather than just along the edge in CZM or classical FEM. However, those authors employed only crack tip enrichments in 2-D and the computational meshes were aligned with the material interfaces, reflecting its weakness. Moreover, simulating new crack formulation onset at the interface by XFEM is challenging. Also, this technique often requires the construction of level-set functions to describe crack, which are often cumbersome for multiple cracks interaction and/or 3-D implementation. Nevertheless, some other approaches

${ }^{*}$ Corresponding author: Tinh Quoc Bui, Associate Professor, Tokyo Institute of Technology, Department of Civil and Environmental Engineering, 2-12-1-W8-22, Ookayama,Meguro-ku, Tokyo 152-8552, Japan. Tel \& Fax:+81 (0)80-5734-2674

e-mail: thanh-tung.nguyen@uni.lu (Thanh-Tung Nguyen), bui.t.aa@m.titech.ac.jp (Tinh Quoc Bui) 
have been also proposed for modeling fracture of interfaces in layered systems such as a FE model for fracture spacing phenomena in layered rocks ([6]), a progressive fracture modeling ([64]) or a discontinuous numerical model for layer/matrix interface properties ([60]).

\subsection{Approach and Objective}

In recent years, the phase field model to fracture mechanics has been emerged, which goes beyond certain limitations of LEFM theory of Griffith ([24]). In phase field model, cracks should propagate along a path of least energy. The brittle fracture is described by a diffuse approximation of discontinuities through an additional phase field variable $([44,3,4])$. In addition to the primary displacements field, this new phase field variable is defined in terms of the energy functional to regularize the geometric discontinuity, or jump, of the displacement, representing the crack. Noticed that the solution of regularized phase field models converges to the solution of sharp crack description implying discontinuities, in the $\Gamma$ - convergence sense $([40,16,17])$. The most advantage of the phase field model is its ability in modeling crack initiation and propagation without any prescriptions of crack geometries. The crucial idea possessed behind the approach is that no additional constitutive rules required by the theory that impose once a crack starts nucleating, propagating or branching. In the phase field approach, the initiation and evolution of cracks emerges by solving the partial differential equations of the model, representing its striking feature, which results in a significant simplification of the implementation, especially in 3-D problems. Different versions of the phase field model have been developed and, more importantly, they can be used to model very complex, multiple crack fronts, branching in both 2D and 3D without ad-hoc numerical treatments, i.e., without fracture criteria ([31, 5, 27, 34, 41, 13, 50, 22, 69, 21]). However, the application of phase field models to study fracture problems in layered media is rather rare in the literature and still open problem. Just a few relevant contributions recently get published, for instance, the analyses of modulus mismatch on crack growth in bioinspired composites [45, 32] or in biomimetic composites [33]. Recently, the finite width of the external surface was introduced into phase field theory of martensitic phase transformations and melting/solidification ([37, 38]). This led to the multiple interesting scale effects in surface-induced phase transformations. However, a detailed investigation on the interfacial effects, or crack-interface interaction, by an effective regularized approach is still missing.

The purpose of this study is to enhance phase field model to fracture in layered heterogeneous structures with a particular focus on the influences of interfacial properties. The effects of both perfect bonded and cohesive interfaces in terms of phase field model, which have previously been developed by the authors ([50,51, 47]), will be considered. To capture the mismatch between different materials at interface region, we thus introduce an regularized interfacial transition zone in the framework of variational approaches. The objective of proposing such a regularize transition zone is to circumvent drawbacks of the sharp-transition models as the modeling challenging caused by the jumps at mismatch material and geometry discontinuity effects can be treated in a straightforward way. Unlike the sharptransition model, the introduced regularized transition zone is much more effective. More importantly, it is particularly suitable for interfaces as its transition zone is smeared out by defining the so-called internal size parameter, which plays the role as regularization representing the actual width of transition zone. Additional purpose of the present contribution is devoted to the better interpretation of fracture behavior such as crack initiation, propagation, branching, delamination, arrest, and penetration at the interface in layered systems by the proposed model.

The primary distinction in this contribution is in the representation of interfaces by the developed regularized interfacial transition zone in terms of phase field context. This new definition essentially resolves the main modeling challenge in crack-interface fracture problems of layered heterogeneous media. In general, the regularized transition zone behaves similarly to the Heaviside function, but in the smeared context instead. By this way, the sharp description zone can also easily be recovered by setting the regularized transition length to be zero. Analogously, this feature of the regularized interfacial transition zone is quite similar to the main concept of the phase field approaches.

The present study focuses on numerical interpretation of mechanical behavior of crack-interface interactions in 2$\mathrm{D}$ and 3-D layered structures under quasi-static load by the proposed model, clarifying the important role of interfacial regularized transition zone. We first study problems of single crack-interface interactions in 2-D and 3-D layered structures, and then apply the proposed method to analyze multiple cracks-interfaces interaction in three-layered 
structures, which is aimed to show its applicability in modeling problems with more complicated configurations. Experimental validation dealing with crack growth in a multi-layered structure is also performed and analyzed. In this paper, we restrict our investigation to consider cracks whose direction is perpendicular to the interfaces only. Here, different types of interfaces, i.e., the perfectly and cohesive bonded interfaces, are considered. We will show in the numerical experiments that, by using the present approach, modeling crack growth and merging in layered media becomes very efficient. We will additionally illustrate one important issue of the present model as it well reproduces the analytical prediction by $[35,36]$. In the subsequent sections, we first derive the variational phase field model to fracture with cohesively bonded interface, describing the regularized interfacial transition zone, and then provide representative numerical examples of single and multiple fracture in 2-D and 3-D layered structures, shedding light on the characteristics of crack paths at the interfaces of layered media.

\subsection{Outline}

The paper begins by stating fundamentals of mechanical characteristics of interfaces in layered media in Section 2, addressing three types of interfaces. In Section 3, we present the concepts of phase field model with a particular focus on the application for layered material. In Section 4, approaches used for description of interfacial zone in layered media are presented, in which a regularized interfacial transition zone is derived. In Section 5, numerical results for single crack-interface interactions in 2-D and 3-D layered systems and multiple cracks with fracture spacing in three-layered structures are analyzed. Experimental validation is also investigated by considering crack growth in a multi-layered structure. Some major conclusions drawn from the study are given in Section 6.

\section{Mechanical characterization of interface in layered media}

It is well known that the mechanical properties of the interface play a crucial role in the fracture study of layered materials and structures $([59,29,26])$. The interface in layered media can be characterized using a variety of different principles. Figure 1 schematically represents an interface in a bimaterial body. In general, it can roughly be divided into three types of interface mainly based on the continuity of mechanical fields: (a) the perfectly bonded interface; (b) the cohesive or the cohesively bonded interface; and (c) the debonded interface.

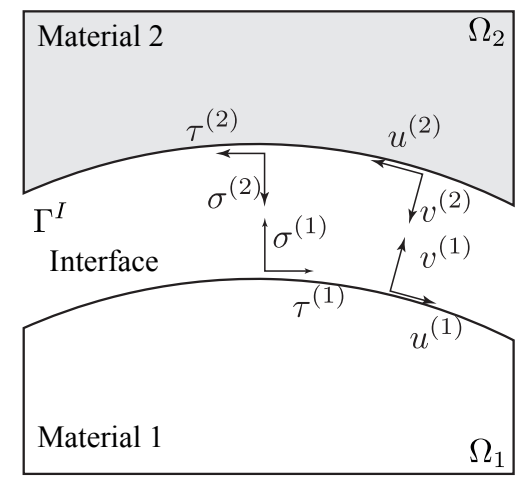

Fig. 1. Description of constitutive relations on the layered material interface. The normal and tangential stresses at the interface are denoted by $\sigma, \tau$, respectively, while the displacement in normal and tangential directions by $u$ and $v$.

The perfectly bonded interface is an infinite strong interface, which can be understood for the case that different layers are bonded by a chemical and/or physical bond. Hence, both the stresses and displacements crossing the interface are assumed to be continuous, i.e., the following relation is established:

$$
\left.\begin{array}{l}
\tau^{(1)}=\tau^{(2)} \\
\sigma^{(1)}=\sigma^{(2)} \\
u^{(1)}=u^{(1)} \\
v^{(1)}=v^{(2)}
\end{array}\right\} \text { on } \Gamma^{I}
$$


The cohesively bonded interface is the one without any physical and/or chemical adhesive bonds between different layers. Therefore, the stresses crossing interface are described by a function of relative displacements (or displacement jump). The most popular cohesive interface model is the one introduced by [46], in which the constitutive relation is defined by

$$
\left.\begin{array}{l}
\sigma^{(1)}=\sigma^{(2)}=t^{\mathrm{n}}(\llbracket u \rrbracket, \llbracket v \rrbracket) \\
\tau^{(1)}=\tau^{(2)}=t^{\mathrm{t}}(\llbracket u \rrbracket, \llbracket v \rrbracket) \\
\text { with } \llbracket v \rrbracket=\left|v^{(1)}-v^{(2)}\right| \text { and } \llbracket u \rrbracket=\left|u^{(1)}-u^{(2)}\right|
\end{array}\right\} \text { on } \Gamma^{I},
$$

where $t^{\mathrm{n}}$ and $t^{\mathrm{t}}$ are the cohesive traction and shear, respectively.

The debonded interface describes an interface that is subjected a loading beyond its finite strength. In this situation the interfacial behavior primarily relates to the kinematics of the interface, such as frictional and contact phenomena. There are a number of models that have been proposed to describe this kind of interface. An example of the simple shear lag model [57,68], where the constitutive relation at the interface is expressed as

$$
\left.\begin{array}{l}
\sigma^{(1)}=\sigma^{(2)} \\
\left|\tau^{(1)}\right|=\left|\tau^{(2)}\right|=f \\
f=-\mu \sigma^{(1)} \text { for }-\sigma^{(1)} \geq 0
\end{array}\right\} \text { on } \Gamma^{I},
$$

where $f$ stands for the friction surface traction and $\mu$ is the frictional coefficient.

In this contribution, we restrict our study by considering only the perfectly bonded interface and the cohesively bonded interface. Then, the fracture phenomena depending on the characterization of each interface will be discussed in detail.

\section{Phase field models for interfaces}

In this section, two phase field models associated with either the perfectly bonded interface and or the cohesively bonded interface are presented. It should be noticed that, in this paper, we focus our attention on exploring physical phenomena of the interfacial fracture problem in layered heterogeneous structures rather than the numerical methodologies. Therefore, other issues such as the accuracy or convergence of the proposed numerical models are not presented here as they have already been demonstrated in the previous works by the present authors, see e.g., Refs. [50, 51, 49] for detail.

\subsection{Classical phase field model with the perfectly bonded interface}

Using a phase field method we study interfacial effects on fracture mechanics of layered structures. We briefly present here the fundamental concepts of the phase field method, and interested readers may refer to, e.g., [41, 50], for detail and its practical implementation aspects. The phase field method is based on a regularized formulation of a sharp crack description. A regularized variational principle describing both the evolution of the mechanical problems and an additional field $d$ describing the damage, which is often called as phase field, with $d=0$ indicating the intact solid, $d=1$ representing the crack, is discretized by a finite element procedure and a staggered algorithm. It is noticed that the staggered algorithm adopted here is not the only choice. Other approaches in general are also possible, for instance, the phase-field model can be solved by using a monolithic scheme, where the displacements and the phase field are solved simultaneously ([34, 42, 67]).

Figure 2 schematically shows smeared cracks in a heterogeneous bi-material $\left(\Omega_{1}\right.$ and $\left.\Omega_{2}\right)$ containing an interface $\Gamma^{I}$ in terms of the phase field framework. Basically, the method alleviates the shortcomings of re-meshing crack geometry by using a fixed mesh and a regularized description of the discontinuities. In addition, crack initiation can be modeled in a straightforward manner.

In the phase field method at small strains, the regularized form of the energy describing the cracked structure is expressed as

$$
E(\mathbf{u}, d)=\int_{\Omega} \psi_{u}(\boldsymbol{\varepsilon}(\mathbf{u}), d) \mathrm{d} \Omega+\int_{\Omega} g_{c} \gamma(d, \nabla d) \mathrm{d} \Omega,
$$




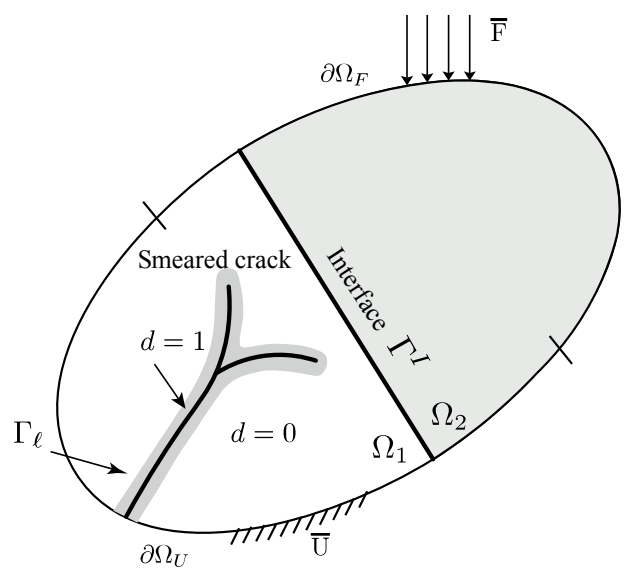

(a) sharp interface

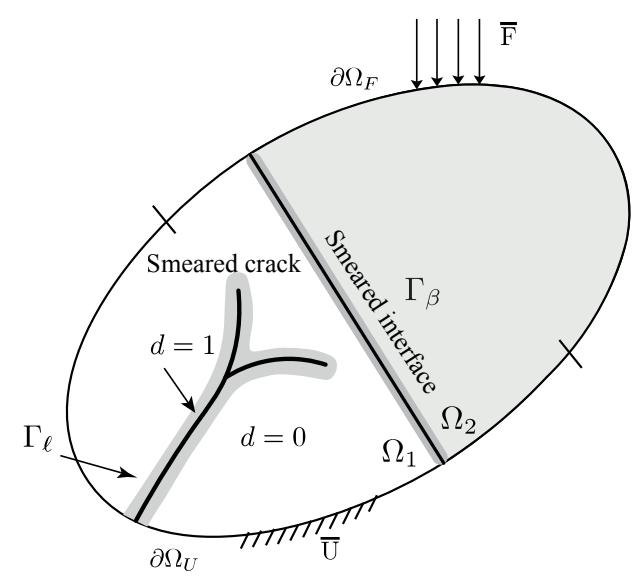

(b) smeared interface

Fig. 2. Problem definition: phase field description of smeared cracks in a heterogeneous bi-material.

where $\varepsilon$ is the linearized strain tensor, while $\psi_{u}$ is the elastic strain energy density, which depends on both the displacements $\mathbf{u}(\mathbf{x})$ and the phase field $d(\mathbf{x})$ describing the damage of solid. $g_{c}$ defines the fracture energy describing the amount of energy dissipated upon the creation of a unit fracture surface. $\gamma(d, \nabla d)$ is the crack density function per unit volume as stated in e.g., [41, 50], given by

$$
\gamma(d, \nabla d)=\frac{1}{2 \ell} d^{2}+\frac{\ell}{2} \nabla d \cdot \nabla d,
$$

in which $\ell$ is the regularization parameter.

It has shown that this kind of regularized model converges to the brittle failure theory when $\ell$ vanishes $[2,15,12]$ as a result of the $\Gamma$-convergence theory. Herein, $\ell$ can be considered as a pure numerical parameter of the regularized model in brittle fracture or thought as a real material parameter for a gradient damage approach. It is recommended in the first case to take $\ell$ as small as possible to better approximate brittle fracture, with regards to the size of the investigated domain. In the second case, $\ell$ however should be identified from the experiment. Such analysis with experimental validations has recently been studied in $[49,48]$.

The total energy can then be rewritten as

$$
E=\int_{\Omega} \psi \mathrm{d} \Omega
$$

with

$$
\psi=\psi_{u}(\varepsilon, d)+g_{c} \gamma(d, \nabla d)
$$

The unilateral contact formulation, by following the work of [41], is chosen to ensure damage induced by traction only, (assuming isotropic elastic behavior of the body) through:

$$
\psi_{u}(\boldsymbol{\varepsilon}, d)=g(d) \psi^{+}(\boldsymbol{\varepsilon})+\psi^{-}(\boldsymbol{\varepsilon}) .
$$

with

$$
\psi^{ \pm}(\boldsymbol{\varepsilon})=\frac{\lambda}{2}\left[\langle\operatorname{tr} \boldsymbol{\varepsilon}\rangle_{ \pm}\right]^{2}+\mu \operatorname{tr}\left[\left(\boldsymbol{\varepsilon}^{ \pm}\right)^{2}\right]
$$

where $\varepsilon^{+}$and $\varepsilon^{-}$are the extensive and compressive modes of strain field $\varepsilon=\varepsilon^{+}+\varepsilon^{-}$. In $(9),\langle x\rangle_{ \pm}=(x \pm|x|) / 2$ Based on the study of $[12,41]$, the simple form of the quadratic degradation function $g(d)$ in Eq. (8) is chosen by $g(d)=(1-d)^{2}+k$. The small parameter $k<<1$ is introduced to maintain the well-posedness of the system for partially broken parts of the domain [12]. 
The evolution of phase field that could guarantee the irreversibility of the process is derived from the thermodynamically consistent framework. A detailed description for that issue is outlined in the Appendix A, and here reads as

$$
2(1-d) \mathcal{H}-\left(d-\ell^{2} \Delta d\right)=0 \text { in } \Omega,
$$

belong with a homogeneous Neumann condition $\nabla d(\mathbf{x}) \cdot \mathbf{n}=0$ on $\partial \Omega$.

The Euler-Lagrange equations of the variational principle for energy minimization to Eq. (4):

$$
\mathbf{u}(\mathbf{x})=\operatorname{Arg}\left\{\inf _{\mathbf{u} \in \mathcal{S}_{u}}\left(E(\mathbf{u}, d)-W^{e x t}\right)\right\},
$$

where $\mathcal{S}_{u}=\left\{\mathbf{u} \mid \mathbf{u}(\mathbf{x})=\overline{\mathbf{u}}\right.$ on $\left.\partial \Omega_{u}, \quad \mathbf{u} \in H^{1}(\Omega)\right\}$ and $W^{\text {ext }}=\int_{\Omega} \mathbf{f} \cdot \mathbf{u} \mathrm{d} \Omega+\int_{\partial \Omega_{F}} \overline{\mathbf{F}} \cdot \mathbf{u} \mathrm{d} \Gamma$ with $\mathbf{f}$ and $\overline{\mathbf{F}}$ being body forces and prescribed traction over the boundary $\partial \Omega_{F}$, yields the mechanical problem defined by

$$
\begin{cases}\nabla \cdot \boldsymbol{\sigma}(\mathbf{u}, d)+\mathbf{f}=\mathbf{0} & \text { in } \Omega, \\ \mathbf{u}(\mathbf{x})=\overline{\mathbf{u}} & \text { on } \partial \Omega_{u}, \\ \boldsymbol{\sigma} \mathbf{n}=\overline{\mathbf{F}} & \text { on } \partial \Omega_{F} .\end{cases}
$$

In Eq. (12), $\boldsymbol{\sigma}=\frac{\partial \psi}{\partial \varepsilon}$ is the second-order Cauchy stress tensor (A.3), $\overline{\mathbf{u}}$ are the prescribed displacement on the boundary $\partial \Omega_{u}$. The constitutive law is expressed as

$$
\boldsymbol{\sigma}=\left[(1-d)^{2}+k\right]\left(\lambda\langle\operatorname{tr} \boldsymbol{\varepsilon}\rangle_{+} \mathbf{1}+2 \mu \boldsymbol{\varepsilon}^{+}\right)+\left(\lambda\langle\operatorname{tr} \boldsymbol{\varepsilon}\rangle_{-} \mathbf{1}+2 \mu \boldsymbol{\varepsilon}^{-}\right)
$$

In the case of the perfectly bonded interface, that requires the continuity for both displacement and interfacial stress (traction). This requirement is simply expressed as

$$
\left.\begin{array}{l}
\left(\sigma^{(1)}-\sigma^{(2)}\right) \mathbf{n}^{\Gamma^{I}}=0 \\
\llbracket u \rrbracket=0
\end{array}\right\} \text { on } \Gamma^{I}
$$

where $\mathbf{n}^{\Gamma^{I}}$ is the unit normal vector field on $\Gamma^{I}$, the notation $\llbracket . \rrbracket$ denotes the jump across $\Gamma^{I}$.

Remark: Apart from the crack density function defined in Eq. (5) above, we here denoted as (G1) for brevity, there are other possible choices available in the literature. For instance, one proposed in $[54,14]$ with $\gamma(d, \nabla d)=\frac{3}{8 \ell} d+\frac{3 \ell}{8} \nabla d$. $\nabla d$ (indicated by G2), which offers some advantages compared to the (G1), such as preventing diffuse damage at small loading and showing a better $\Gamma$-convergence characteristic. However, this model seems to be more computationally expensive (i.e., significant increase of the computational time for direct/indirect solving FEM equations of phase field problem, requiring more iterations for Newton-Raphson method in the coupling with cohesive zone model). Although the (G1) model shows the damage right after loading, the damage value is quasi small. This can be negligible in the global behavior of the material and structure. Hence, the G1 formulation is kept for this study. One solution for such problem could be pursued in future studies, for instance, is to combine the $(\mathrm{G} 2)$ formulation with the higher-order phase-field $\gamma(d, \nabla d, \Delta d)$ introduced in [11], which is noticed to improve the accuracy and convergence of the effective critical energy release rate and the strain energy.

\subsection{Phase field model with the cohesively bonded interface}

In cohesive interface model, it is assumed that only relative displacements across interface can activate stress transfer at the interface. The interface traction (or/and shear) is then modeled as a function of the displacement jump. This definition can introduce the normal tensile (or/and shear) stress degradation with increasing the displacement discontinuity. Recently, the cohesive fracture has been incorporated into the phase field/gradient damage models in several contributions, e.g., $[18,66,51,25,52]$, which opens a new door to investigate the interfacial cracking phenomena. 
In order to account the behavior of the cohesively bonded interface into phase field model, a new energy term is added to describe the cohesively bonded interface $\psi^{I}(\llbracket \mathbf{u} \rrbracket, \kappa)$, which depends on the displacement jump $\llbracket \mathbf{u} \rrbracket$ across the interface $\Gamma^{I}$, and $\kappa$ is a history parameter to distinguish between loading and unloading. Once the cohesive fracture $\psi^{I}$ is determined, the cohesive tractions are derived through its differentiation, for more detail, see e.g., [47].

$$
\mathbf{t}(\llbracket \mathbf{u} \rrbracket, \kappa)=\frac{\partial \psi^{I}(\llbracket \mathbf{u} \rrbracket, \kappa)}{\partial \llbracket \mathbf{u} \rrbracket}
$$

The regularized description for strong discontinuities related to interfaces is also adopted. In other words, the sharp interface is regularized by a smear interface (substituting $\Gamma^{I}$ by $\left.\Gamma_{\beta}=\int_{\Omega} \gamma_{\beta}(\beta, \nabla \beta) \mathrm{d} \Omega\right)^{1}$, i.e., the interfacial energy will be regularized by $\gamma_{\beta}(\beta, \nabla \beta)$, see Fig. 2(b). Further, the displacement jump $\llbracket \mathbf{u} \rrbracket$ created by interfacial decohesion is approximated as a smooth transition $\mathbf{v}(\mathbf{x})$. The two approaches have already been proposed and reported in our previous work [47], one based on a Taylor expansion at first order of the assumed smoothed regularized displacement field, and the other used an addition field coupled in the boundary value problems. The advantages and drawbacks of each method have been discussed in [47]. In the present work, to the aim of numerical stability, the second method is adopted. It is worth mentioning that the proposed approach is highly suitable for investigating complex interfaces and be able to deal with the arbitrary geometries of interfacial region.

The infinitesimal strain tensor can be now decomposed into a part related to the bulk (elastic part) and a part induced by the smoothed jump at the interface (inelastic part), denoted by $\boldsymbol{\varepsilon}^{e}$ and $\tilde{\boldsymbol{\varepsilon}}$, respectively

$$
\varepsilon=\varepsilon^{e}+\tilde{\varepsilon},
$$

and the inelastic part can be defined following the works $[66,51]$

$$
\tilde{\boldsymbol{\varepsilon}}=\mathbf{n}^{\Gamma_{\beta}} \otimes^{s} \mathbf{v} \gamma_{\beta} .
$$

with $\mathbf{n}^{\Gamma_{\beta}}$ being the normal vector to $\Gamma_{\beta}$, which can be directly determined from the smeared interface field, as follows

$$
\mathbf{n}^{\Gamma_{\beta}}(\mathbf{x})=\frac{\nabla \beta(\mathbf{x})}{\|\nabla \beta(\mathbf{x})\|} .
$$

The strain energy of a damageable material is here replaced by $\psi_{u}^{e}\left(\boldsymbol{\varepsilon}^{e}, d\right)$. The above functional Eq. (4) is rewritten as

$$
E=\int_{\Omega} \psi_{u}^{e}\left(\boldsymbol{\varepsilon}^{e}, d\right) \mathrm{d} \Omega+\int_{\Omega} g_{c} \gamma(d, \nabla d) \mathrm{d} \Omega+\int_{\Omega} \psi^{I}(\mathbf{v}, \kappa) \gamma_{\beta}(\beta, \nabla \beta) \mathrm{d} \Omega
$$

This definition implies that for $\beta(\mathbf{x}) \rightarrow 0$ (away from the interface) then $\gamma_{\beta} \rightarrow 0$ and $\boldsymbol{\varepsilon}^{e} \rightarrow \boldsymbol{\varepsilon}$, we recover the regularized energy functional for brittle fracture without interfaces as Eq. (4).

The density of the total energy now is expressed as

$$
\psi=\psi_{u}^{e}\left(\varepsilon^{e}, d\right)+g_{c} \gamma(d, \nabla d)+\psi^{I} \gamma_{\beta} .
$$

The new evolution law for phase field variable can now be thermodynamically constructed in the same manner as the Appendix A, which yields

$$
2(1-d) \mathcal{H}^{e}-\left(d-\ell^{2} \Delta d\right)=0 \text { in } \Omega, \text { with } \nabla d(\mathbf{x}) \cdot \mathbf{n}=0 \text { on } \partial \Omega,
$$

and the history function $\mathcal{H}^{e}(\mathbf{x}, t)$ is here expressed the same as the Eq. (A.13), but for elastic strain $\varepsilon^{e}$ (see [51, 47] for more details).

\footnotetext{
${ }^{1}$ The smeared interface is here described in the same manner as Eq. (5), by introducing $\gamma_{\beta}(\beta, \nabla \beta)=\frac{1}{2 \ell_{\beta}} \beta(\mathbf{x})^{2}+\frac{\ell_{\beta}}{2} \nabla \beta(\mathbf{x}) \cdot \nabla \beta(\mathbf{x})$, for $\ell_{\beta} \rightarrow 0$ this description leads to the exact representation of the sharp cohesive interface (see e.g., [51, 47] for more detail.)
} 
Considering the quasi-static equilibrium equations without body forces, the corresponding equations describing the mechanical problem are derived as

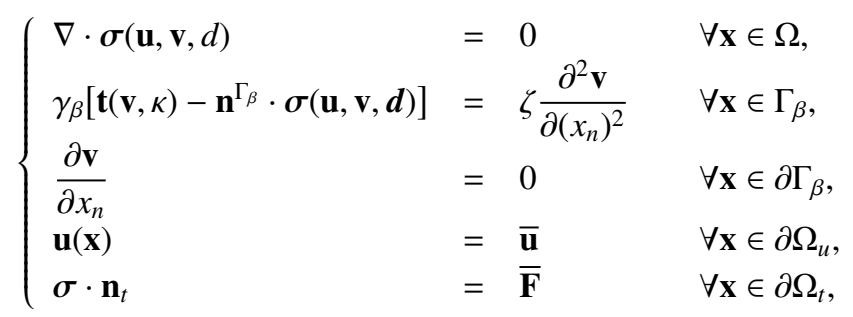

where $x_{n}=\left(\mathbf{x}-\mathbf{x}_{\Gamma^{I}}\right) \cdot \mathbf{n}^{\Gamma^{I}}$ and $\mathbf{x}_{\Gamma^{I}}=\underset{\mathbf{y} \in \Gamma^{I}}{\operatorname{argmin}}(\|\mathbf{y}-\mathbf{x}\|)$, the positive constant $\zeta$ is introduced to ensure that the auxiliary displacement jump field is constant in the normal direction. $\mathbf{t}(\mathbf{v}, \kappa)$ are the tractions at the interfaces introduced in Eq. (15), with outward normal $\mathbf{n}_{\Gamma^{l}}$; and $\overline{\mathbf{F}}, \overline{\mathbf{u}}$ are the prescribed tractions and prescribed displacements on $\partial \Omega_{F}, \partial \Omega_{u}$, respectively.

Clearly, within the context of this approach, the phase field $d$ describes the bulk crack surface density, as well as the interface crack density, allowing interaction between both crack types in an efficient manner. The advantages of cohesive zone model is benefited, such as the complex interfacial behavior can be accurately described using a mixed mode cohesive law, accounted the strength effects of interface in both normal and tangential directions. Moreover, the numerical analysis in [51] demonstrated that the solution is convergent with respect to the mesh and the crack path is mesh-independent, making a significant improvement compared to the classical CZM.

\subsection{Numerical implementation}

In general, Eqs. (10), (12), (22), (21) are solved by a standard FE procedure with a staggered scheme at each load increment. More theoretical and practical details can be found e.g., [41, 50, 47].

\section{Description of interfacial zone in layered materials}

We discuss in this section existing and new approaches that are used for modeling material properties at interfacial region in layered structures. To this end, we introduce a regularized transition based on an internal characteristic length which is highly suitable for capturing such interfacial zone. The material properties of bi-material layered structure at interfacial region can be described by a classical way sharp transition, i.e., the interfacial size is here taken to be null, which is thus expressed as follows:

$$
\begin{cases}\phi=0 & \forall \mathbf{x} \in \Omega_{1} \\ \phi=1 & \forall \mathbf{x} \in \Omega_{2}\end{cases}
$$

where $\phi(\mathbf{x})$ is the material characteristic.

Alternatively, such interfacial zone could also be modeled through a regularized transition by introducing a transition region, say $\Omega_{\text {Trans }}$, with an internal characteristic size, which is indicated here as $\ell_{\text {Trans }}$ :

$$
\begin{cases}\phi=0 & \forall \mathbf{x} \in \Omega_{1} \\ 0<\phi<1 & \forall \mathbf{x} \in \Omega_{\text {Trans }} \\ \phi=1 & \forall \mathbf{x} \in \Omega_{2} .\end{cases}
$$

In this study, the regularized transition zone is constructed as the same underlying principle of the phase field method, which is by first solving the following boundary value problem (BVP) on $\Omega$.

$$
\begin{cases}\phi-\ell_{\text {Trans }}^{2} \Delta \phi=0 & \text { in } \Omega, \\ \phi(\mathbf{x})=1 & \text { on } \Gamma^{I}, \\ \nabla \phi(\mathbf{x}) \cdot \mathbf{n}=0 & \text { on } \partial \Omega,\end{cases}
$$




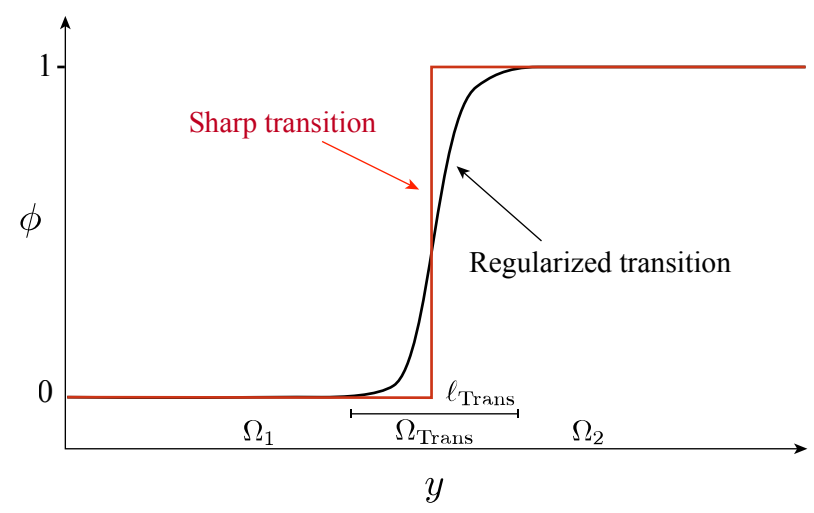

Fig. 3. Schematic of different approaches for describing an interface region: the classical sharp transition and the regularized one.

and then

$$
\phi(\mathbf{x})= \begin{cases}\frac{1}{2} \phi(\mathbf{x}) & \forall \mathbf{x} \in \Omega_{1}, \\ \frac{1}{2}(2-\phi(\mathbf{x})) & \forall \mathbf{x} \in \Omega_{2},\end{cases}
$$

where $\Delta($.$) is the Laplacian, the internal size \ell_{\text {Trans }}$ plays the role as a regularization parameter describing the actual width of the transition zone, and $\mathbf{n}$ is the outward normal to $\partial \Omega$. The BVP yields the solution as described in Eq. (24). The one-dimensional illustration of both descriptions of the transition zone is schematically depicted in Fig. 3. Interestingly, it can be seen in Fig. 3 that the sharp transition behaves similarly to the Heaviside function, but the regularized transition smears out and its variation is fully dependent on the internal characteristic size. In other words, the behavior of regularized transition zone is controlled by the internal characteristic size, revealing its major distinction with respect to the classical sharp transition approach.

By introducing the interpolation function, which satisfies $h(\phi=0)=0, h(\phi=1)=1$ and $\left.\frac{\partial h(\phi)}{\partial \phi}\right|_{\phi=0, \phi=1}=0$; one choice: $h(\phi)=-2 \phi^{3}+3 \phi^{2}$. The material parameter such as the Young's modulus $E$, and fracture energy $g_{c}$ in the whole domain can then be approximated by

$$
\left\{\begin{array}{l}
E=[1-h(\phi)] E^{(1)}+h(\phi) E^{(2)} \\
g_{c}=[1-h(\phi)] g_{c}^{(1)}+h(\phi) g_{c}^{(2)},
\end{array}\right.
$$

where $E^{(1)}, g_{c}^{(1)}$ and $E^{(2)}, g_{c}^{(2)}$ are the Young's modulus and fracture energy for material M1 and material M2, respectively. Noted that the interpolations described in Eq. (27) are to ensure that $E=E^{(1)}$ in $\Omega_{1}, E=E^{(2)}$ in $\Omega_{2}$, and smooth transition from $E^{(1)}$ to $E^{(2)}$ in the interfacial zone.

For simplicity, we restrict our study in this paper by considering three types of interface modeling, which are detailed as follows:

- (C1) Sharp transition and perfect interface

- (C2) Sharp transition and cohesive interface

- (C3) Regularized transition and perfect interface.

Remark: The other case is the regularized transition and cohesive interface, which has not been considered and treated in this paper. It is because that, according to our observation, the sudden changes in material properties are the origin of the discontinuity in the displacement, providing the cohesive properties of the interface. However, since the regularized transition does not provide the displacement jump, the combination of the regularized transition and cohesive interface is thus not the case. 


\section{Representative numerical examples and experimental validation}

In this section, the interfacial effects on fracture behavior of layered structure are numerically investigated. We first consider two-layered structures containing an initial crack as shown in Fig. $4^{2}$. For better interpretation of the numerical results, both 2-D and 3-D models for the two-layered structures are thus analyzed. To this end, results of crack propagation calculated by the proposed approach are presented and discussed. The effects of material mismatch on fracture behavior of layered structures are also explored. To avoid the size effects on the behavior of fracture, the size of layer here is thus chosen the same. Secondly, to show the applicability of the proposed approach, examples with more complex configurations such as multiple crack growths in three-layered structures are then considered (see Fig. 17). Finally, crack propagation in a multi-layered structure as depicted in Fig. 24 is studied, which serves the purpose of experimental validation.

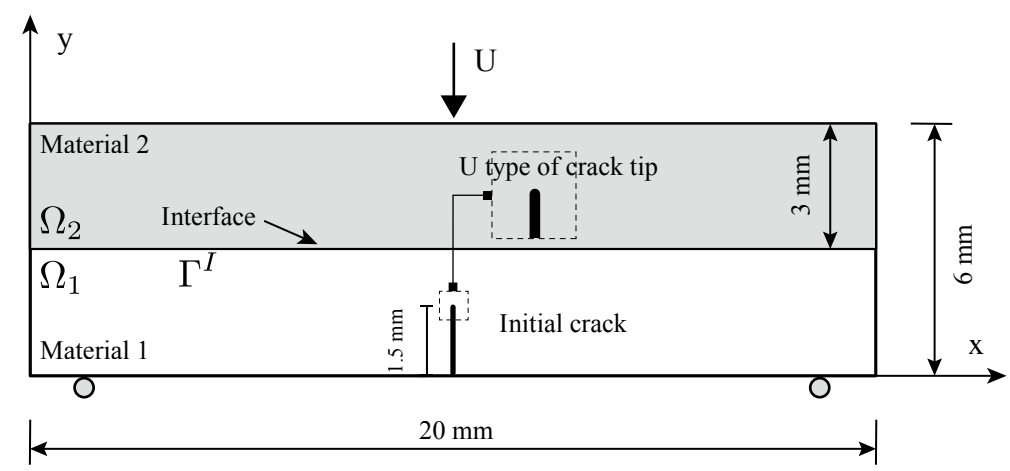

(a) 2D model

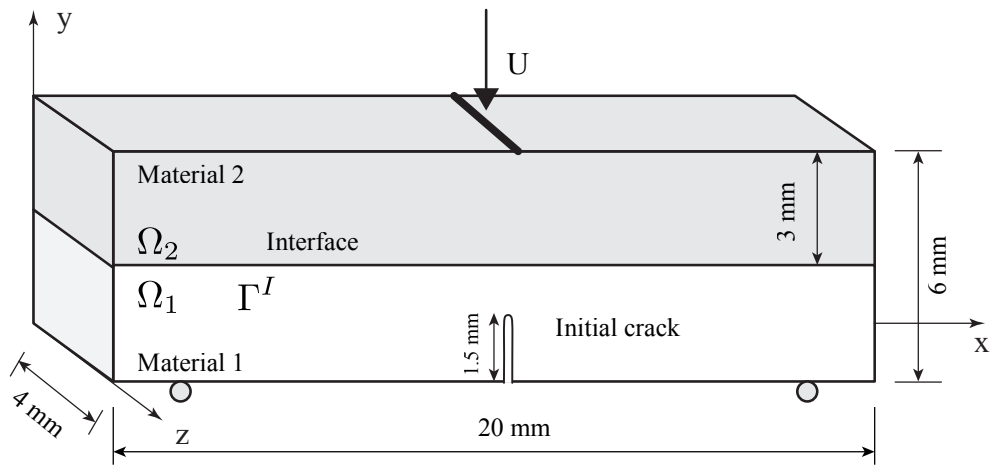

(b) 3D model

Fig. 4. Schematic of bi-material layered structures containing cracks and their geometry and boundary conditions for (a) 2-D model and (b) 3-D model. The material information used for these two layered structures and other relevant parameters are detailed in the numerical results section.

In the numerical implementation, an FE model using a fixed mesh with a refined mesh around crack paths has been used, where the characteristic size $h_{\min }$ is taken for the region of expected crack path and larger elements whose size are $h_{\max } \mathrm{mm}$ have been employed away from initial crack. The details of those parameters are described in Table 1. It should be noted that the mesh used here is fine enough (compared to the internal length) to obtain a converged solution.

\footnotetext{
${ }^{2}$ In the phase field model, the length scale should be larger than the characteristic length of structure to alleviate the mesh effects. Hence, the U-type of initial crack tip is chosen in all numerical examples to ensure this condition.
} 


\begin{tabular}{|c|c|c|c|}
\hline Model & $h_{\min }[\mathrm{mm}]$ & $h_{\max }[\mathrm{mm}]$ & Number of elements \\
\hline 2-D & 0.025 & 0.075 & 3124435 \\
3-D & 0.035 & 0.1 & 5335212 \\
\hline
\end{tabular}

Table 1. Parameters description of FE refined meshes in 2-D and 3-D models

\subsection{Influences of material mismatch on fracture of layered materials}

This study aims to analyze the effects of material mismatch on fracture behavior of layered structure by the present phase field method. The 2-D model described in Fig. 4(a) is taken for the analysis. The sharp transition and perfect interface $(\mathrm{C} 1)$ is considered. More specifically, we investigate the influences of mismatch ratio between material M1 and material M2 on the crack crossing interface. To this aim, the elastic modulus, the Poisson's ratio and fracture energy for the first material (M1) is fixed as $E^{(1)}=12 \mathrm{GPa}, v^{(1)}=0.3, g_{c}^{(1)}=1.4 \times 10^{-6} \mathrm{kN} / \mathrm{mm}$, while those for the second material (M2) are taken as

$$
\left\{\begin{array}{l}
E^{(2)}=f_{m} E^{(1)} \\
v^{(2)}=v^{(1)}=0.3 \\
g_{c}^{(2)}=f_{m} g_{c}^{(1)}
\end{array}\right.
$$

where $f_{m}$ is defined as the mismatch ratio, and various specific values of the ratio, e.g., $f_{m}=[1,2,3,3.5,5,8,10]$, are considered, respectively.

The crack regularized parameter $\ell=0.1 \mathrm{~mm}$ is selected for the analysis. The plane strain condition is assumed. The monotonic compressive displacement $\Delta U=-5 \times 10^{-5} \mathrm{~mm}$ is prescribed as long as the phase field $d<0.8$ in all elements, and $\Delta U=-1 \times 10^{-5}$ as soon as $d \geq 0.8$ in one integration point, in the total 800 increments $^{3}$. The crack paths obtained for different mismatch ratios computed by the present model are then shown in Fig. 5 .
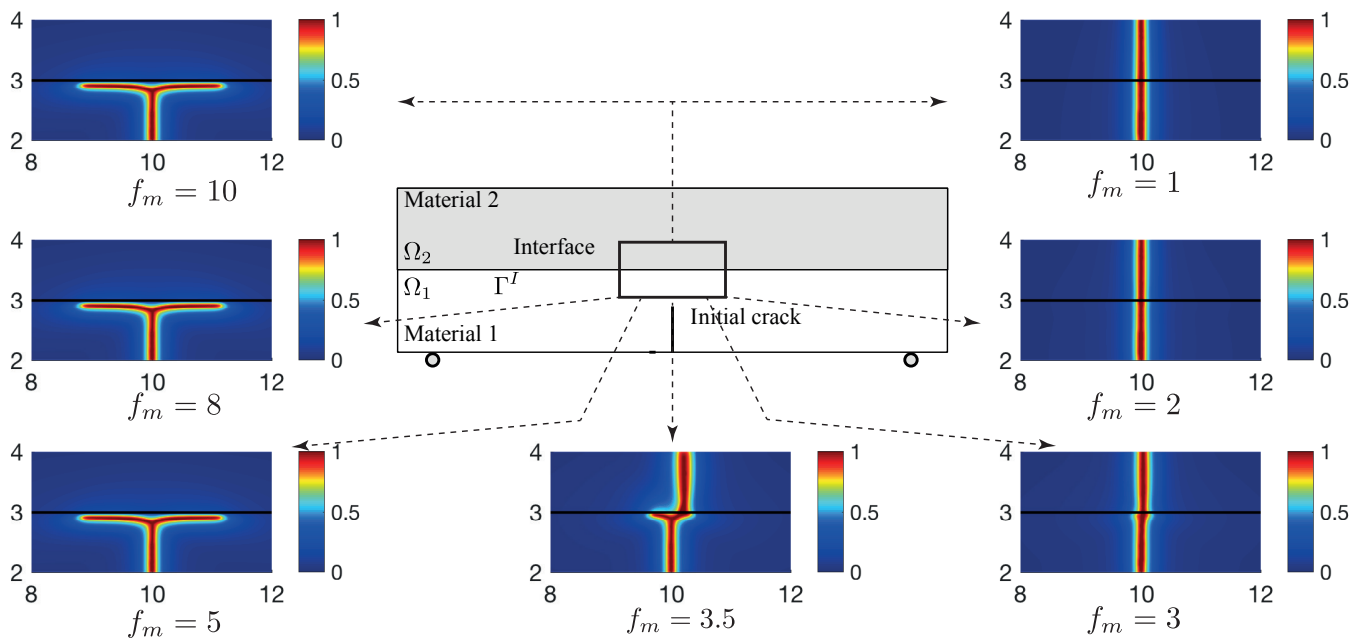

Fig. 5. Crack paths for different given values of the mismatch ratio at the same loading $U=-0.0086$ mm, obtained by the present model. The mismatch ratio greatly alters the fracture behavior at the interface in layered structure. One important issue can be seen that increasing layered contrast leads to the crack branching at the interface.

The obtained numerical results of crack paths clearly show a strong dependence of fracture behavior (i.e., crack penetration, branching) on the characteristics of material mismatch. Through these results, it is obvious that the material mismatch ratio significantly alters the fracture phenomena at the interfaces in layered structures. The discrepancy

\footnotetext{
${ }^{3}$ The staggered scheme seems to be dependent on incremental loading size. It could provide the problem of overestimation of the amount of dissipation energy for large incremental loading size [67, 19]. Therefore, a small incremental value is encouraged to obtain a converging solution.
} 
in the fracture behavior at the interfaces is easily understood. For the first case $f_{m}=1$, the homogeneous structure is recovered, and no interfacial effects are observed. However, the situation changes as slight effects have been seen from the results that have low contrast between two layers, e.g., see the crack path by $f_{m}=2,3$. In this case, the crack can penetrate the interface quite easily. This phenomenon is restricted when the layered mismatch increases. As observed from, for instance, $f_{m}=3.5$, where the crack starts unsymmetrically branching at the interface, and for a while it propagates a bit along the interface, then the crack grows into the second layer even at low loading condition. On the contrary, obtained results of cracks for other cases of the mismatch ratios, e.g., $f_{m}=5,8,10$ do not show similar situations as the aforementioned cases. At such a loading condition, the cracks for the larger cases of the mismatch ratio are not able to penetrate the interfaces, or to be arrested by the interface, the cracks branching are found instead. This would be potential properties required for the application of FGM materials. Loosely speaking, to ensure well performance of layered structures, the layered contrast must be taken rather five times. This value is consistent with the one recently reported by $[35,36,32]$.

\subsection{Influences of interfacial properties on fracture of layered materials}

The effects of interface strength on the failure mode of layered heterogenous material are important and their numerical investigation is thus presented here. By accomplishing it, the 2-D model described in Fig. 4(a) is again considered using the sharp transition and cohesive interface model $(\mathrm{C} 2)$. The material mismatch ratio defined in Eq. (28) is taken as $f_{m}=2$. This chosen value ensures the fact that the crack can easily penetrate into the second material if the delamination mode is not favorable, i.e., it makes the influences of interface strength on the failure mode more significantly.

In the cohesive model, for the sake of simplicity, only mode-I interface law is considered in this analysis. The interface cohesive energy is taken in accordance to the work of [70]:

$$
\psi^{I}(\llbracket u \rrbracket)=g_{c}^{I}\left[1-\left(1+\frac{\llbracket u \rrbracket}{v_{n}}\right) \exp \left(-\frac{\llbracket u \rrbracket}{v_{n}}\right)\right] .
$$

The normal traction law is obtained as

$$
t(\llbracket u \rrbracket)=g_{c}^{I} \frac{\llbracket u \rrbracket}{v_{n}^{2}} \exp \left(-\frac{\llbracket u \rrbracket}{v_{n}}\right),
$$

where $v_{n}=g_{c}^{I} /\left(t_{u} \exp (1)\right) ; t_{u}$ is the fracture strength.

The fracture energy for interface is taken as $g_{c}^{I}=f_{i} g_{c}^{(1)} \mathrm{kN} / \mathrm{mm}$, where $f_{i}$ can be considered as the mismatch ratio of fracture resistance between interface and bulk material. Various specific values of the mismatch, e.g., $f_{i}=\left[\begin{array}{llll}0.01 & 0.1 & 1 & 10\end{array}\right]$, are considered. The obtained results of crack morphology are then illustrated in Fig. 6. A switching from purely delamination mode to bulk failure mode is clearly captured. The weak interfaces are favorable to the delamination behavior, while the strong interface resulted in the dominant bulk failure mode. The observed phenomena are consistent with the existing studies in literature.

\subsection{Fracture at low loading phase: $2-D$ model}

We aim to study the mechanical characterization of interface on the fracture behavior of 2-D layered structures under a low loading condition, considering three models of the interface $(\mathrm{C} 1),(\mathrm{C} 2)$ and $(\mathrm{C} 3)$. The elastic stiffness $E^{(2)}$, and fracture energy $g_{c}^{(2)}$ of material M2 is taken five times larger than those of material M1. The material parameters and other relevant model inputs for the present bi-material structures are detailed in Table 2. In essence, this choice is to avoid the crack crossing the interface in the classical situation (i.e., (C1) perfectly bonded interface sharp transition) as described in the previous subsection 5.1. Therefore, we expect that the fracture phenomena will be influenced by only the interface behavior.

In the second case $(\mathrm{C} 2)$, the mode I described in subsection 5.2 is considered in this test. The fracture strength is $t_{u}=1 \mathrm{MPa}$ and the fracture energy for interface is taken $g_{c}^{I}=10^{-6} \mathrm{kN} / \mathrm{mm}$. 


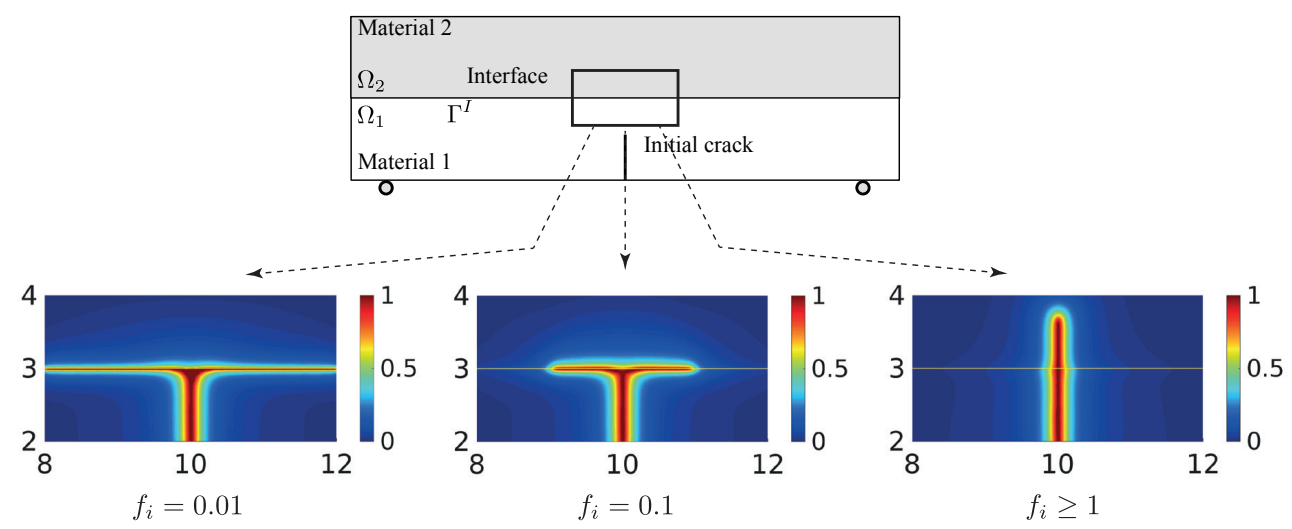

Fig. 6. Crack paths for different given values of the mismatch ratio $f_{i}$ of fracture resistance between interface and bulk material at the same loading $U=-0.008 \mathrm{~mm}$, obtained by using sharp transition and cohesive interface model. The increase of interface strength resulted by switching the failure mode from interfacial delamination to bulk fracture.

\begin{tabular}{|c|l|l|l|l|}
\hline Material & $E(\mathrm{GPa})$ & $v$ & $g_{c}(\mathrm{kN} / \mathrm{mm})$ & $\ell(\mathrm{mm})$ \\
\hline M1 & 12 & 0.3 & $1.4 \times 10^{-6}$ & 0.1 \\
M2 & 60 & 0.3 & $7 \times 10^{-6}$ & 0.1 \\
\hline
\end{tabular}

Table 2. Two layered structure: material parameters

Fig. 7 schematically shows the variation of the Young's modulus $E$ in the line of investigation by both the sharp and regularized descriptions of interface. For the regularized transition, different values of $\ell_{\text {Trans }} / \ell$ varying from 0.75 to 2.5 are considered, which is to exhibit the characteristics and variation of the regularized transition size in the present model. It can easily be seen in the figure that the smaller the $\ell_{\text {Trans }}$ approaches zero, the regularized transition converges to the sharp one, and once $\ell_{\text {Trans }}=0$ both descriptions are identical. Unlike the classical sharp model, those discussed features clearly reveal the more flexibility of the regularized interfacial transition approach.

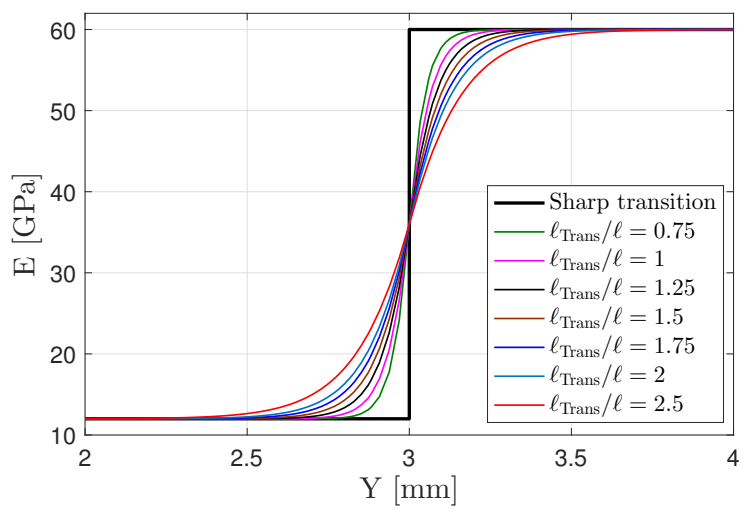

Fig. 7. Transition field across the interface on a line of investigation.

In this analysis, the monotonic compressive displacements increments prescribed on the top edge of the specimen with $U=-5 \times 10^{-5} \mathrm{~mm}$ as long as the phase field $d<0.8$ in all elements, and $\Delta U=-1 \times 10^{-5}$ as soon as $d \geq 0.8$ in one integration point, in the total 800 increments are taken. The plane strain condition is also assumed. Our initial investigation is devoted to the interpretation of displacement behavior that crosses the interface. In particular, the displacement along a line of investigation (e.g., $x=10 \mathrm{~mm}$ ) in the $y$ - direction at loading step $U=0.0002$ 
$\mathrm{mm}$ is considered, and its computed results are then shown in Fig. 8(a). Different states of the displacement are depicted in the numerical results. The sharp and regularized transitions with perfect interface provide the continuity of displacement when passing from $\Omega_{1}$ to $\Omega_{2}$. At the interface $\Gamma_{I}$, a sudden change of the displacement is captured by the sharp transition shown in Fig. 8(b), whereas a smooth change is obtained by the regularized transition. We can further observe here that the increase the internal length $\ell_{\text {Trans }}$ the smoother the displacements are obtained, and more interestingly, it converges to the sharp description of interface when $\ell_{\text {Trans }} \rightarrow 0$. The sharp transition with cohesive interface however exhibits different behavior as compared to the previous ones. Here, the jump of the displacement between two materials is clearly observed in the pictures. In the smeared context, this phenomenon was analytically described in [35].

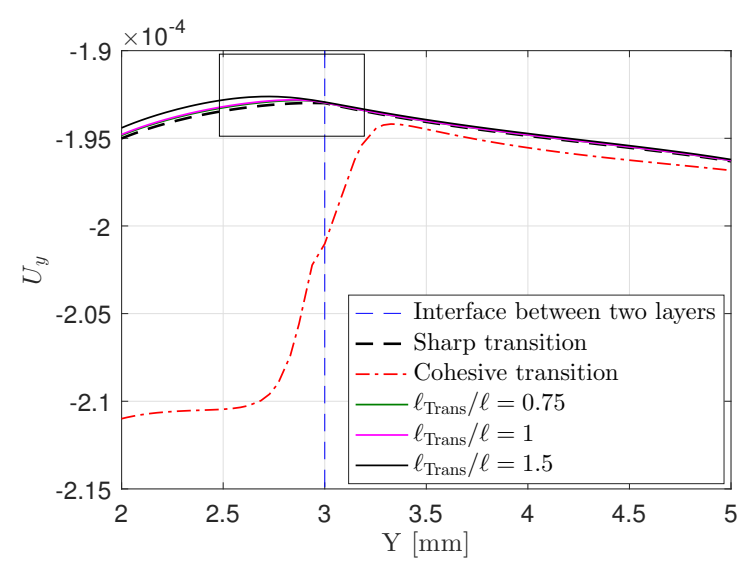

(a) Region near interface

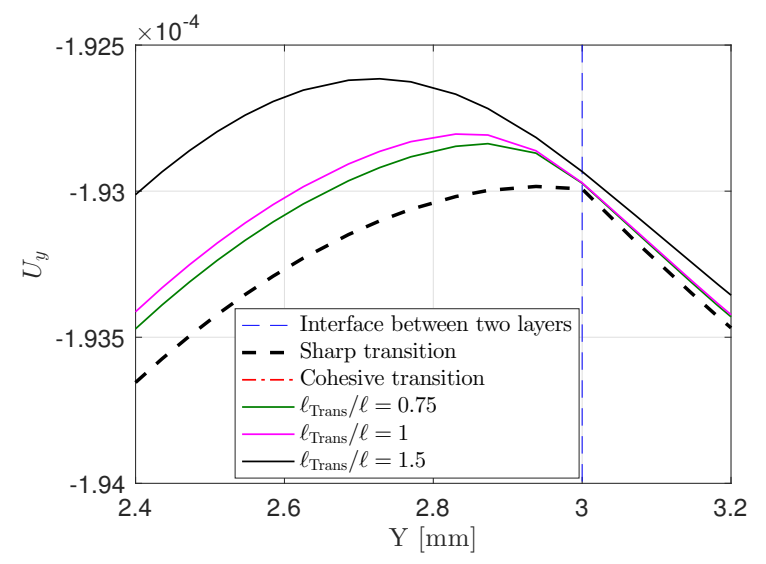

(b) Zoom at the interface transition

Fig. 8. Displacement in $y$-direction on the line of investigation. Sharp transition stands for the (C1) while Cohesive transition for (C2).

The comparison of crack path for different considered situations is depicted in Figs. 9, 10 and 11. The first case (C1) in Fig. 9, the sharp transition and perfect interface, shows the propagation of crack along the interface after reaching this region and no crack onset in $\Omega_{2}$. The sudden change of material properties between two layers (and $g_{c}^{(2)}>g_{c}^{(1)}$ ) provides a gap in critical fracture energy $g_{c}$. Therefore, a large amount of the strain energy is needed in order to initiate crack in the second layer. However, according to our assumption, the stiffness of the second material is stronger than that of the first one (i.e., $E^{(2)}>E^{(1)}$ ). In this scenarios, the second layer must be deformed less than the first layer at interface region (see Fig. 8). With the same contrast $\frac{E^{(2)}}{E^{(1)}}=\frac{g_{c}^{(2)}}{g_{c}^{(1)}}$, the strain energy in the second layer under the low loading is not enough to form crack (see Fig. 12(a)). The same phenomenon is captured in the case (C2), the interfacial delamination after crack reaches the interface as shown in Fig. 9. 


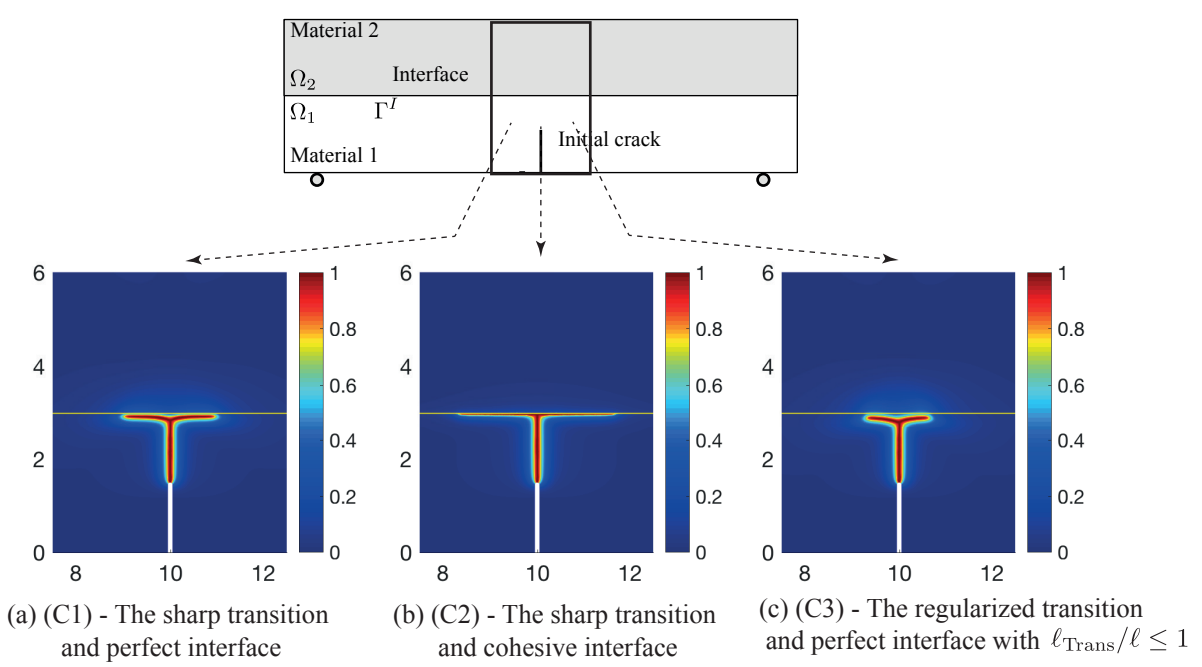

Fig. 9. Analysis of transition size on fracture behaviour: comparison of crack path among three approaches.

The fracture phenomenon is different for the case (C3) as shown in Fig. 9, wherein the smooth transition zone is added between two layers. A strong dependence on the size of the transition zone is captured. Based on our own numerical experiments, different values of $\ell_{\text {Trans }}$ yield different crack paths. When the regularized transition length $\ell_{\text {Trans }}$ is set to be smaller than or equal to the crack regularized length $\ell$, i.e., $\ell_{\text {Trans }} / \ell \leq 1$, the crack also propagates along the interface after reaching the transition zone, i.e., branching. However, as shown in Fig. 10, the distance from the point at which crack merges to the interface is different between (C3) and (C1). The regularized transition zone makes the interface having thickness (equal twice the regularized transition length $2 \ell_{\text {Trans }}$ ), hence that moves the crack merging a bit in comparison with the sharp case.

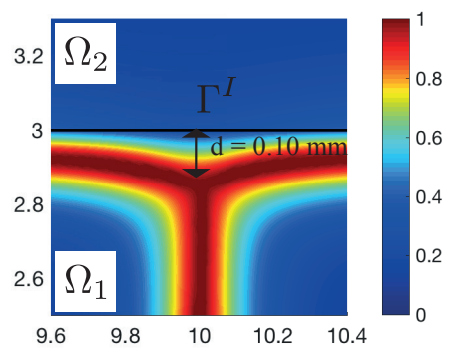

(a)

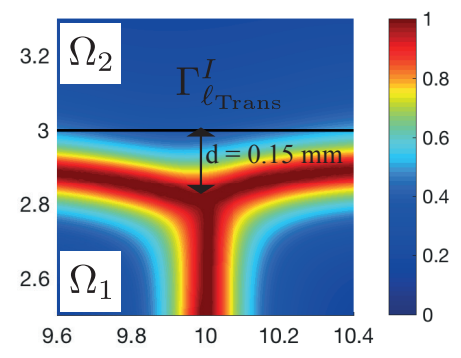

(b)

Fig. 10. Comparison of crack path between two cases: (a) The sharp transition and perfect interface - (C1); (b) The regularized transition and perfect interface $-(\mathbf{C 3})$ with $\ell_{\text {Trans }} / \ell=1$.

Another phenomenon can also be captured when the regularized transition length $\ell_{\text {Trans }}$ is assumed to be greater than the crack regularized length $\ell$. Fig. 11(a) shows a bit of crack merging at the transition zone but the crack then propagates to the second material. When $\ell_{\text {Trans }} / \ell \geq 1.5$, the crack is fully across the transition zone with a slight influence of the interface. No crack branching holds.

To look at more insight into the effects of transition zone on the fracture phenomena, the strain energy and fracture energy in a line of investigation along initial crack $(x=10)$ are hence analyzed. Their obtained results are depicted in Fig. 12. Noted that the crack creation by means of the phase field method is controlled by the ratio between the positive part of strain energy $\psi^{+}(\mathbf{x})$ and fracture energy $g_{c}$. A material point will be cracked, provided that the following condition $\psi^{+} / g_{c} \gg 1$ must be satisfied. In the case of sharp transition (see Fig. 12(a)) the sudden gap 


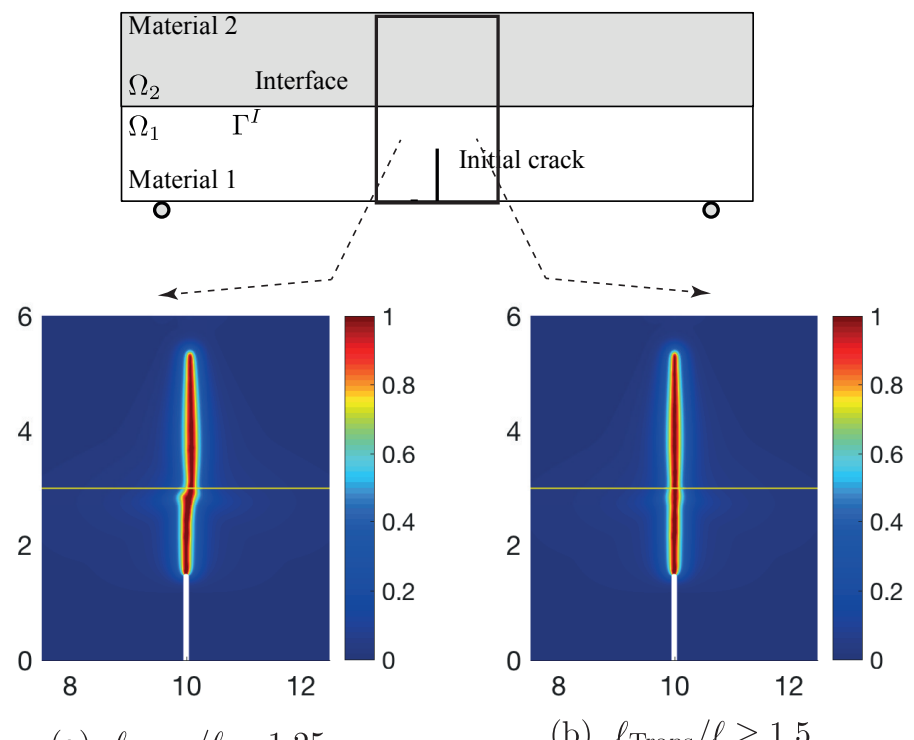

(a) $\ell_{\text {Trans }} / \ell=1.25$

(b) $\ell_{\text {Trans }} / \ell \geq 1.5$

Fig. 11. Effects of the transition size on fracture behavior: comparison of crack path between two cases of the regularized transition.

within the material parameters leads to a large change of the displacement between two layers (see Fig. 8), inducing also a sudden gap of the strain energy. When the ratio $\frac{E^{(2)}}{E^{(1)}}=\frac{g_{c}^{(2)}}{g_{c}^{(1)}} \geq 5$, the strain energy produced at the interface is not sufficient for crack propagating in the second layer. However, the smooth transition zone creates the thickness interface, where the strain energy transmits smoothly from layer to layer (see Fig. 12(b)). Once increasing $\ell_{\text {Trans }}$, the more energy the transmission from the first layer to the second one is obtained. It means that, while keeping the critical fracture energy, it is expected the propagation of the crack in the second layer when increasing the strain energy $\psi^{+}(\mathbf{x})$ at the interface region.

In summary, with the mismatch ratio $f_{m}=5$, weak interface, the sharp transition - perfect/cohesive interfaces $(\mathrm{C} 1,2)$ exhibit the dominant delamination failure mode, while the regularized transition interface (C3) shows a converging from purely bulk failure to interfacial delamination. Furthermore, in order to quantitatively obverse how the structural stress-displacement responses of the specimens under consideration look like, and how the defined interface models affect the behavior of those global responses, we show the structural stress-displacement responses during the entire loading history for all considered models of interfaces including (C1), (C2) and (C3) with various values of $\ell_{\text {Trans }} / \ell$ in Fig. 13. As can be seen from the picture that the second peak of the curves at approximately $U=5.5-7 \times 10^{-3} \mathrm{~mm}$ are caused by the interface, and the behavior of the curves are fully dependent on the models defined for the interface. At low loading level, the regularized transition (C3) with $\ell_{\text {Trans }} / \ell \leq 1$ (e.g., $\left.0.75,1\right)$ yields quite similar behavior with the sharp $(\mathrm{C} 1)$ and cohesive $(\mathrm{C} 2)$ transitions. For those cases, the behavior of the structural stress-displacement curves is reasonable, as already shown in Fig. 9, the cracks are arrested as they branch along the interface. So no penetration into the second layer is obtained. However, when $\ell_{\text {Trans }} / \ell \geq 1$, the cracks obtained from the regularized transition (C3) penetrate into the second layer (see Fig. 11) and continue to grow until the structures get completely destroyed at much lower stress. At high loading level, the deviations of (C1), (C2) and (C3) with $\ell_{\text {Trans }} / \ell \leq 1$ have been captured. The cohesive interface shows its best resistance, followed by sharp transition interface and regularized transition interface exhibited its lowest resistance. Again, the convergence of mechanical response of regularized transition to sharp transition when $\ell_{\text {Trans }} \rightarrow 0$ is observed.

\subsection{Fracture at high loading phase: comparison between 2-D and 3-D models}

The numerical results shown in the last subsection 5.3 demonstrate the high dependence of crack growth on the interfacial properties. Specially, in the case of perfectly bonded - sharp transition interface $(\mathrm{C} 1)$, the crack propagates 


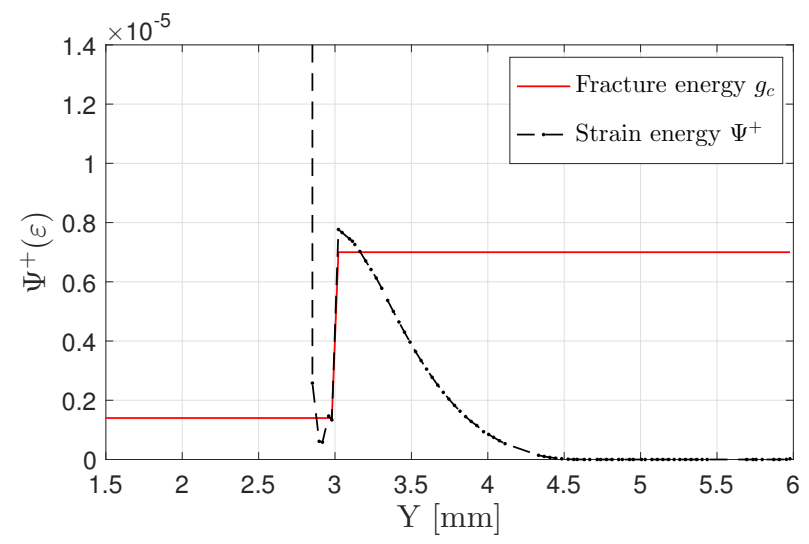

(a) (C1) - The sharp transition and perfect interface

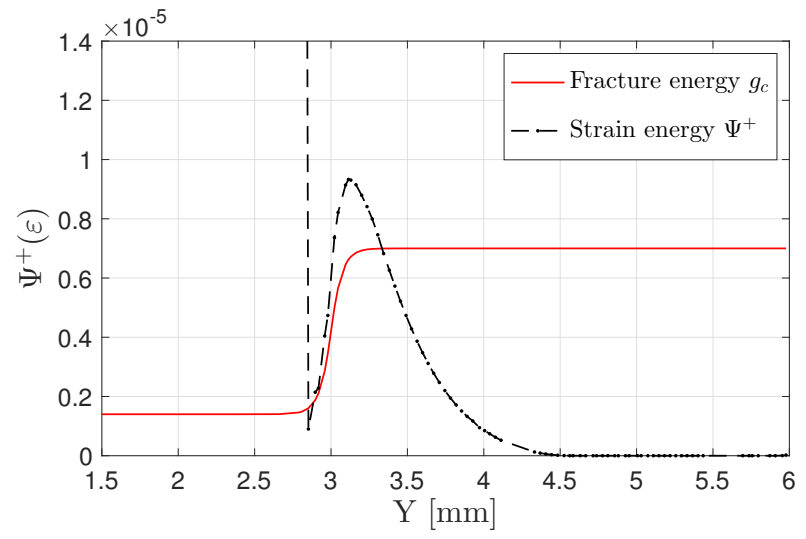

(b) (C3) - The regularized transition with $\ell_{\text {trans }} / \ell=1$

Fig. 12. Schematic of fracture and strain energies on a line of investigation. The strain energies are plotted at the same time step as that one depicted in Fig. 9 .

along the interface after reaching this region. This phenomenon may be significantly different when the structure is subjected to very high loading. To explore this issue more detail, we thus perform numerical simulations on both 2-D and 3-D models at high loading condition. The fully 3-D model also allows to gain more insight into the interfacial fracture behavior in layered structures. For simplicity, and as stated above, only the sharp transition and perfect interface $(\mathrm{C} 1)$ is considered and analyzed.

In the numerical computations, monotonic compressive displacements increments are again prescribed in a line on the top edge of the specimen, with $U=-5 \times 10^{-5} \mathrm{~mm}$ for phase field $d<0.8$, and $\Delta U=-1 \times 10^{-5}$ as soon as $d \geq 0.8$, in the total 1600 increments. The computed results of crack propagation for different loading are depicted in Figs. 14 and 15, respectively. In the first loading phase $\left(U=0:-1.2 \times 10^{-2} \mathrm{~mm}\right)$, the fracture behavior of fully 3-D model is almost similar to that observed in the 2-D model: the crack propagates in the first layer until reaching the interface (see Fig. 14(a)), then it propagates along the interface (see Fig. 14(b)). In Fig. 14(c), we capture a famous geometry of semi-elliptical surface crack, and it seems to symmetrically propagate in both directions $(-x$ and $+x)$ at the interface.

In the second loading phase $\left(U=-1.240 \times 10^{-2}:-1.250 \times 10^{-2} \mathrm{~mm}\right)$, we capture the evolution of crack crossing the interface but different phenomena between 2-D and 3-D simulations are obtained. In the 3-D model, the onset of crack in the second layer occurs at both surface $(x=9.5, z=0$ and $x=10.5, z=4 \mathrm{~mm})$ of the specimen, always in the semi-elliptical form. In the 2-D model, although the onset is quite similar to 3-D model, two cracks appeared in the concentration regions can be seen in the second layered (at interface region, $x=9.5$ and $x=10.5$ ), the crack then crosses the interface to second layer only at left half $(x=9.5)$. 


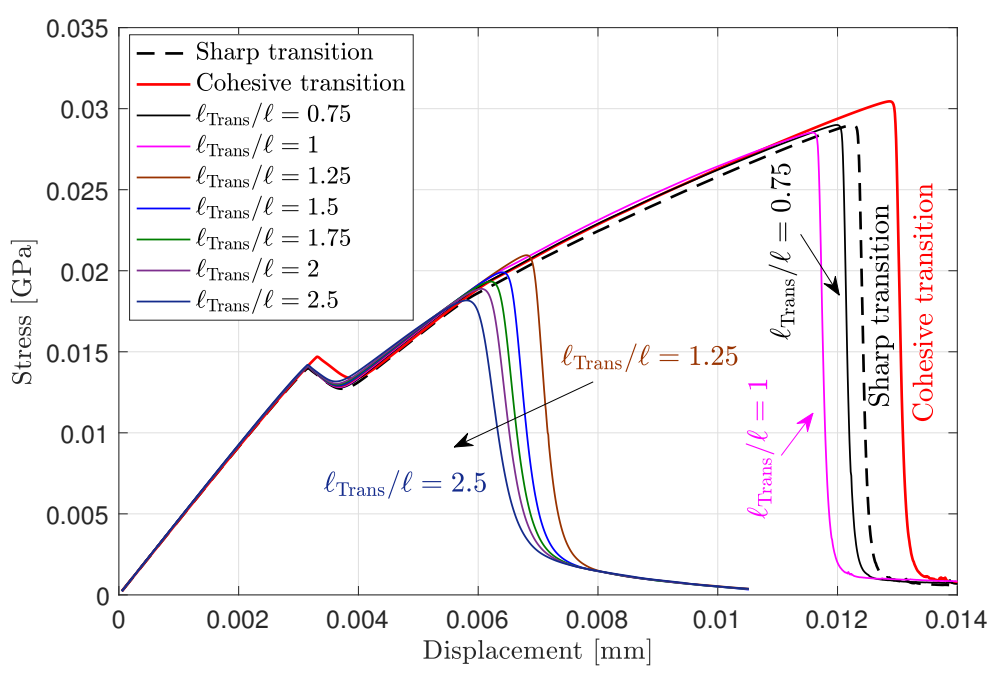

(a) Global load-displacement curves

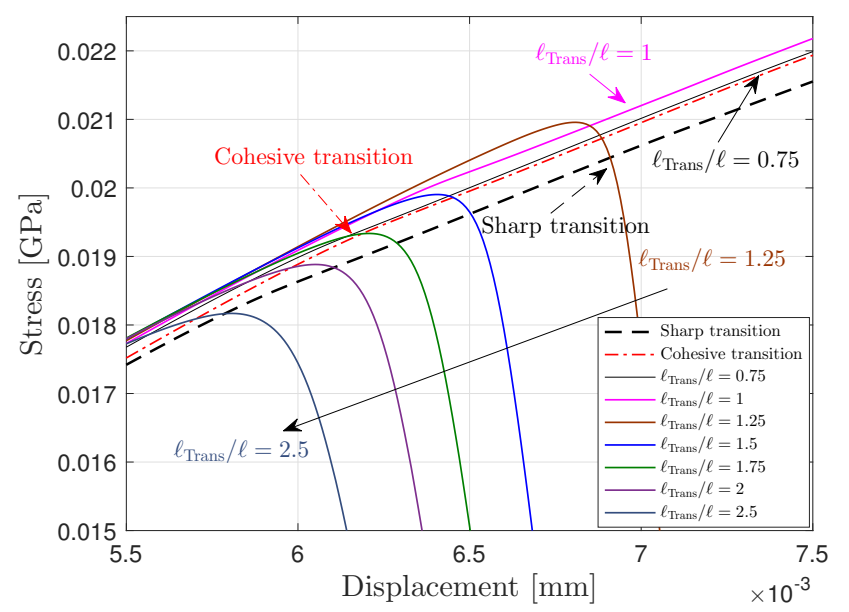

(b) A close-up view at a displacement range from $5.5 \times 10^{-3}$ to $7.5 \times 10^{-3} \mathrm{~mm}$

Fig. 13. Effects of the transition size on the macroscopic load-displacement responses.

At a larger loading, the symmetric geometry of the crack path in the second layer in the 3-D model loses. Instead, a curved crack is captured but mostly at the right half. The crack shown at the structure face $z=4$ is similar to that obtained from 2-D model as depicted in Fig. 16.

\subsection{Multiple cracks: Fracture spacing in three-layered structures}

This example aims to show the applicability of the present phase field approach to crack propagation in complex configurations of layered structures. We thus conduct a three-layered structure with equally spacing and parallel cracks in 2-D as sketched in Fig. 17. This can also be considered as multiple cracks in layered structure. The 2D plane strain simulation is assumed. Special attention is devoted to the cracks location where four equally spacing cracks are setup to be in parallel to the interfaces, and are perpendicular to the applied load. One should be noticed that modeling crack propagation in such multiple cracks structures would be very challenging to the discrete approaches such as the XFEM, e.g., see [73] and references therein. The phase field approach, on the other hand, is home to the effective modeling techniques of these complex structures. In this section, we will show how the developed phase field method is able to effectively simulate complex problem of multiple cracks in three-layered structures. 

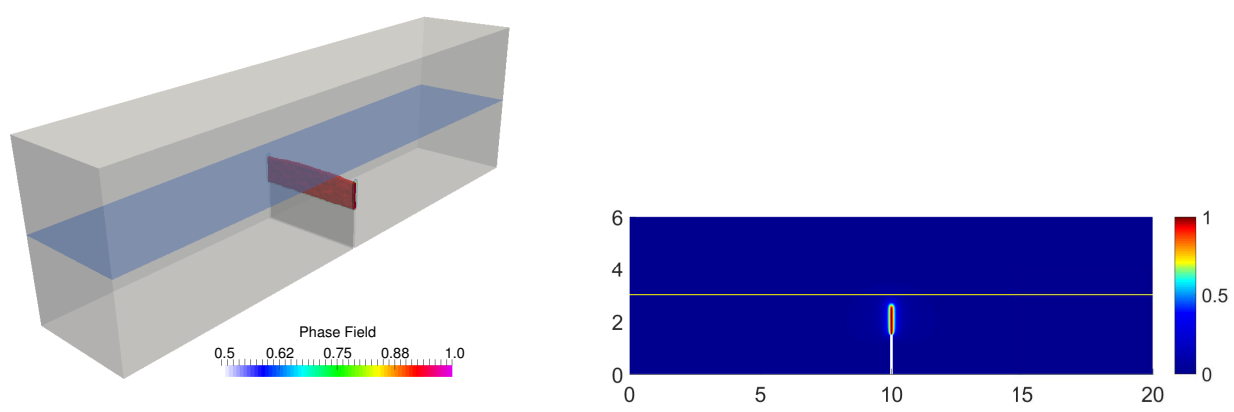

(a) Crack path corresponds to $U=-3.80 \times 10^{-3} \mathrm{~mm}$
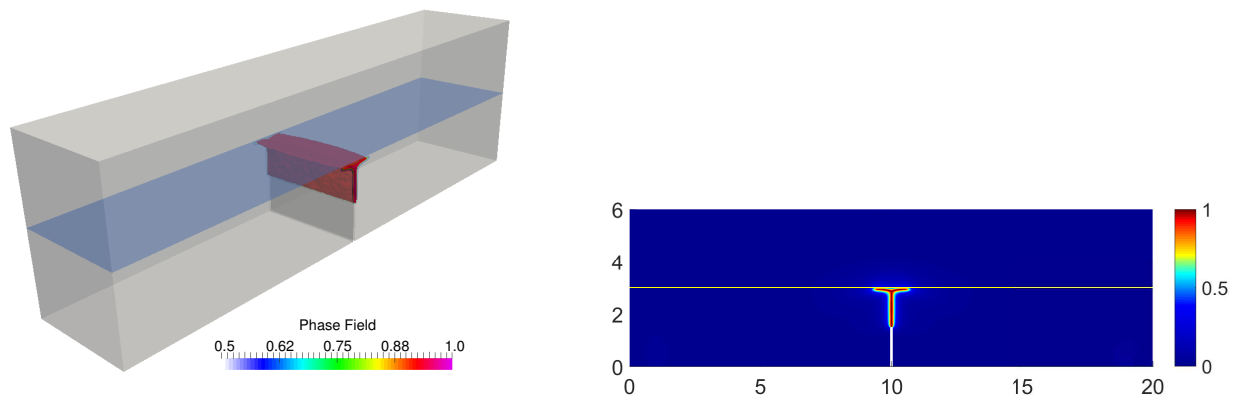

(b) Crack path corresponds to $U=-9.00 \times 10^{-3} \mathrm{~mm}$
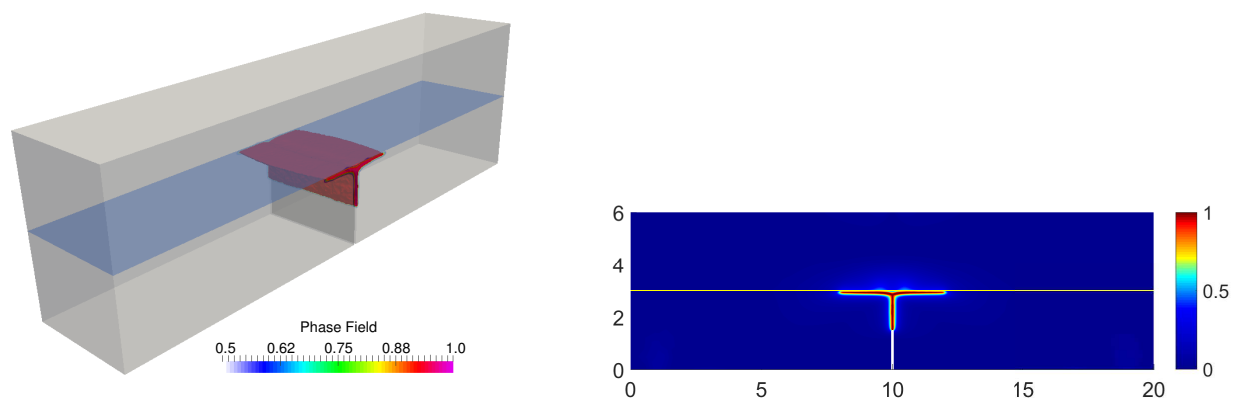

(c) Crack path corresponds to $U=-1.200 \times 10^{-2} \mathrm{~mm}$

Fig. 14. Crack propagation in $2 D$ and $3 D$ layered structures, the iso-surface of phase field $d(x)>0.95$ is plotted for different loading.

The material parameters used for simulation are described in Table 3. For simplicity, we restrict our analysis here to a particular case wherein the second material (the top and bottom layers) is setup to be weaker than the first material (middle layer) with $E^{(2)}=E^{(1)} / 5$, but the fracture energy for the middle layer however is much larger than the other outer layers, i.e., $g_{c}^{(2)}=10 g_{c}^{(1)}$, (see eg. [64] for more detail).

\begin{tabular}{|c|l|l|l|l|}
\hline Material & $E(\mathrm{GPa})$ & $v$ & $g_{c}(\mathrm{kN} / \mathrm{mm})$ & $\ell(\mathrm{mm})$ \\
\hline Material 1 & 50 & 0.25 & $5 \times 10^{-6}$ & 0.1 \\
Material 2 & 10 & 0.35 & $5 \times 10^{-5}$ & 0.1 \\
\hline
\end{tabular}

Table 3. Material parameters used for the three-layered structure depicted in Fig. 17

Similarly, all phase field simulations here are conducted using an FE refined fixed mesh, where the characteristic size $h_{\min }=0.025 \mathrm{~mm}$ is taken for the region of expected crack path and larger elements whose sizes are $h_{\max }=$ $0.5 \mathrm{~mm}$ are employed away from initial crack. The final refined fixed mesh eventually reaches 369870 triangular 

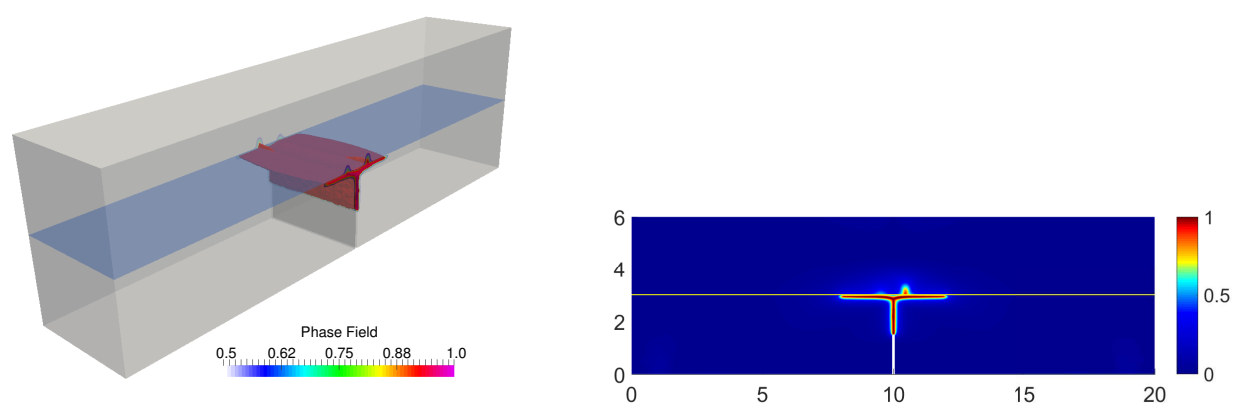

(a) Crack path corresponds to $U=-1.240 \times 10^{-2} \mathrm{~mm}$
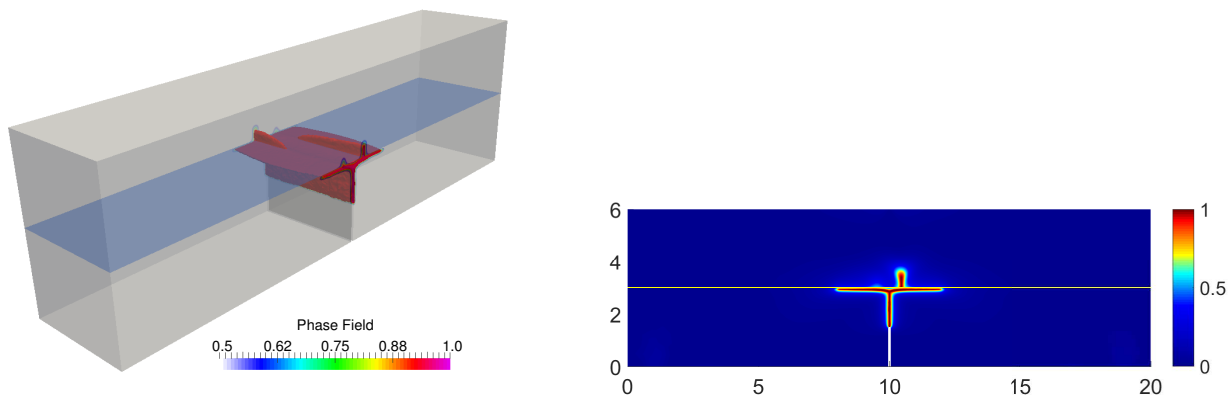

(b) Crack path corresponds to $U=-1.245 \times 10^{-2} \mathrm{~mm}$
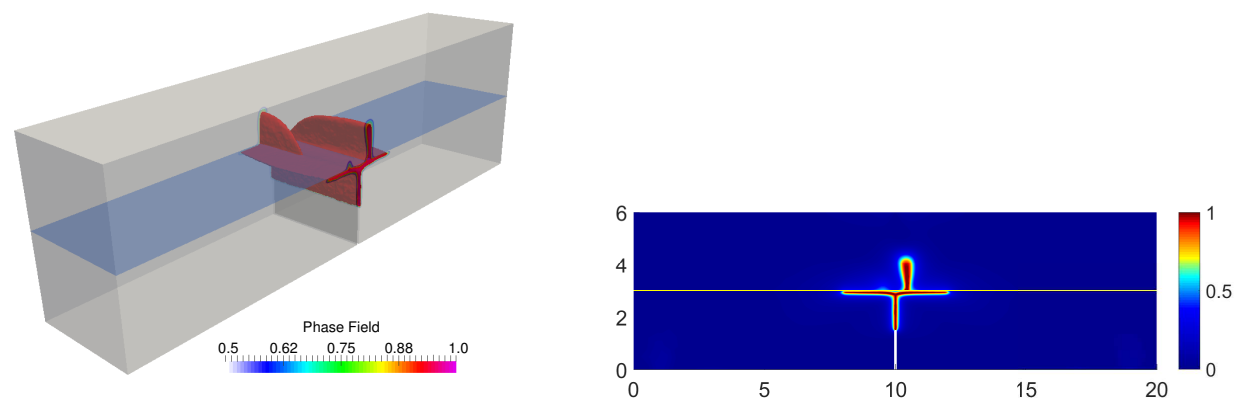

(a) Crack path corresponds to $U=-1.250 \times 10^{-2} \mathrm{~mm}$

Fig. 15. Crack propagation in 2D and 3D layered structures, the iso-surface of phase field $d(\mathbf{x})>0.95$ is plotted for different loading.

elements. The monotonic compressive displacement increments are prescribed on the top edge of the specimen, with $U=1 \times 10^{-4} \mathrm{~mm}$ as long as the phase field $d<0.8$ in all elements, and $\Delta U=-2 \times 10^{-5}$ as soon as $d \geq 0.8$ in one integration point, in the total 1600 increments.

All three models of the interfaces (C1), (C2) and (C3) described above are considered for these multiple cracks in three-layered structures. As compared with the classical case (C1) (see the discussion in subsection 5.3), here the regularized length $\ell_{\text {Trans }}=2 \ell$ is taken for the smooth transition situation (C3) instead, which aims to provide new behavior for interfacial fracture. In the cohesive interface behavior (C2), both normal and tangential components for the cohesive law are considered. The general form in $2 \mathrm{D}$ is given by

$$
\mathbf{t}(\mathbf{w}, \boldsymbol{\alpha})=\left[t^{n}, t^{t}\right]^{\mathrm{T}}
$$

where $t^{n}$ and $t^{t}$ are the normal and tangential parts of the traction vector $\mathbf{t}$ across the interface $\Gamma^{I}$ oriented by its normal $\mathbf{n}^{I}$. A nonlinear elastic cohesive model that is independence on the history is used [20,66]:

$$
t^{n}=g_{c}^{I} \frac{\llbracket u \rrbracket^{n}}{v^{n}} \exp \left(-\frac{\llbracket u \rrbracket^{n}}{v^{n}}\right) \exp \left(-\frac{\left(\llbracket u \rrbracket^{t}\right)^{2}}{\left(v^{t}\right)^{2}}\right),
$$




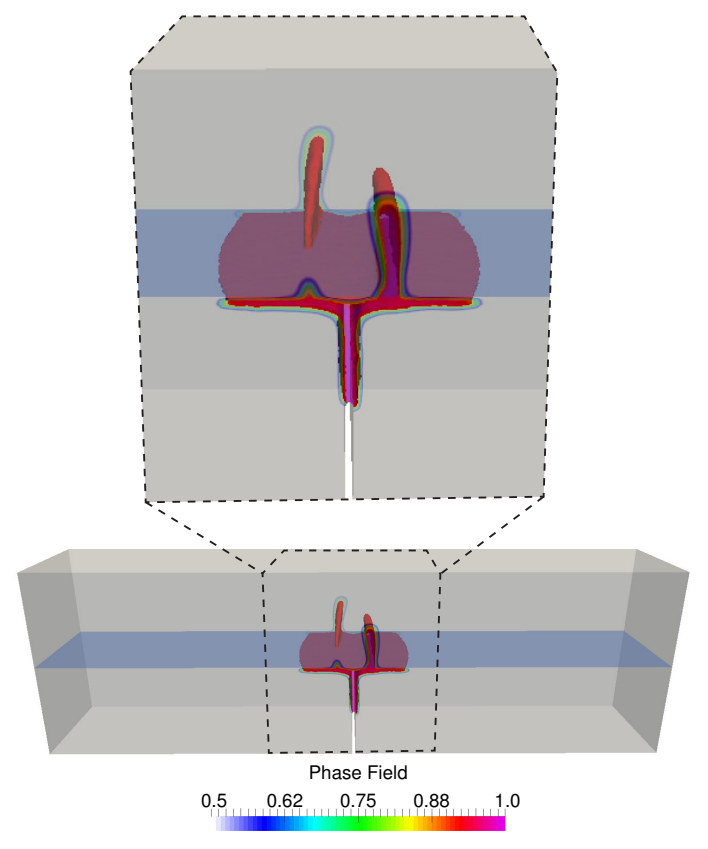

Fig. 16. Crack path crossing second layer in 3-D model

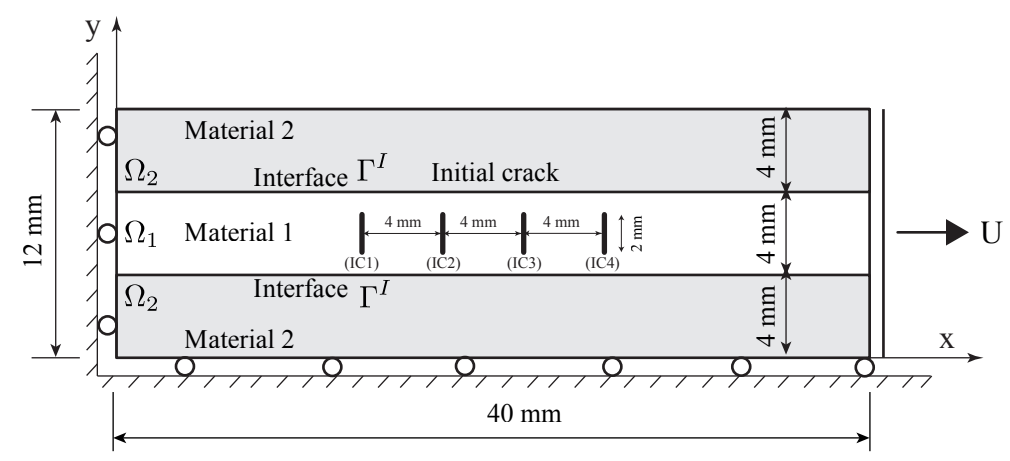

Fig. 17. Schematic of geometry and boundary conditions for a three-layered structure with spacing parallel cracks

where $\llbracket u \rrbracket^{n}=\llbracket u \rrbracket \cdot \boldsymbol{n}^{I}$ and $\llbracket u \rrbracket^{t}=\llbracket u \rrbracket \cdot \boldsymbol{m}^{I}$, with $\mathbf{m}^{I}$ being a tangent vector to $\Gamma^{I}, g_{c}^{I}$ is the toughness associated with the interface, and

$$
t^{t}=2 g_{c} \frac{\llbracket u \rrbracket^{t}}{v^{t}}\left(1+\frac{\llbracket u \rrbracket^{n}}{v^{n}}\right) \exp \left(-\frac{\llbracket u \rrbracket^{n}}{v^{n}}\right) \exp \left(-\frac{\left(\llbracket u \rrbracket^{t}\right)^{2}}{\left(v^{t}\right)^{2}}\right) .
$$

With the critical normal/tangential separation are $v_{n}=g_{c}^{I} /\left(t_{u}^{n} \exp (1)\right)$ and $v_{t}=g_{c}^{I} /\left(t_{u}^{t} \exp (1)\right)$, respectively. The normal/tangential fracture strength are $t_{u}^{n}=1 \mathrm{MPa}$ and $t_{u}^{t}=1.5 \mathrm{MPa}$, The fracture energy for interface is taken $g_{c}^{I}=10^{-6} \mathrm{kN} / \mathrm{mm}$ for both components.

Fig. 18 first shows the computed result of crack propagation for the classical situation (C1). The loss of material resistance is observed at crack onset (e.g., $U=0.0083 \mathrm{~mm}$ ) within the central layer. As clearly shown in Fig. 18 at $U=0.016 \mathrm{~mm}$, the two outer initial cracks (IC1) and (IC4) propagate much faster than the other inner ones, (IC2) and (IC3). The discrepancy may be due to the effects of the boundary conditions. Since the (IC1) and (IC4) locate closely to the simply support and applied loading boundaries, and they may gain more stronger effects than the inner cracks (IC2) and (IC3). 
The behavior of crack propagation for this complex structure becomes more complicated after the two outer initial cracks reaching the interfaces. These two outer cracks stay at the central layer and have not been able to penetrate the outer layers, which enables the transfer of energy from (IC1) and (IC4) to the other inner cracks (IC2), precluding the penetration. The top and bottom layers hence still resist. Moreover, the assumed perfect interface could transmit the loading capacity from the outer layers into the central one, exciting the growing of the two inner initial crack (IC2) and (IC3) rather than the delamination at the interfaces. Consequently, the total loss of resistance of the central layer is obtained at $U=0.027 \mathrm{~mm}$ (see Fig. 18), and beyond this range of applied loading, the penetration of the outer cracks (IC1) and (IC4) into the top and bottom layers is found. At $U=0.032 \mathrm{~mm}$, the structure gets destroyed as sketched in Fig. 18. In general, under such a condition, the structure gets damage in terms of the onset of multiple cracks, not that of the delamination among layers.

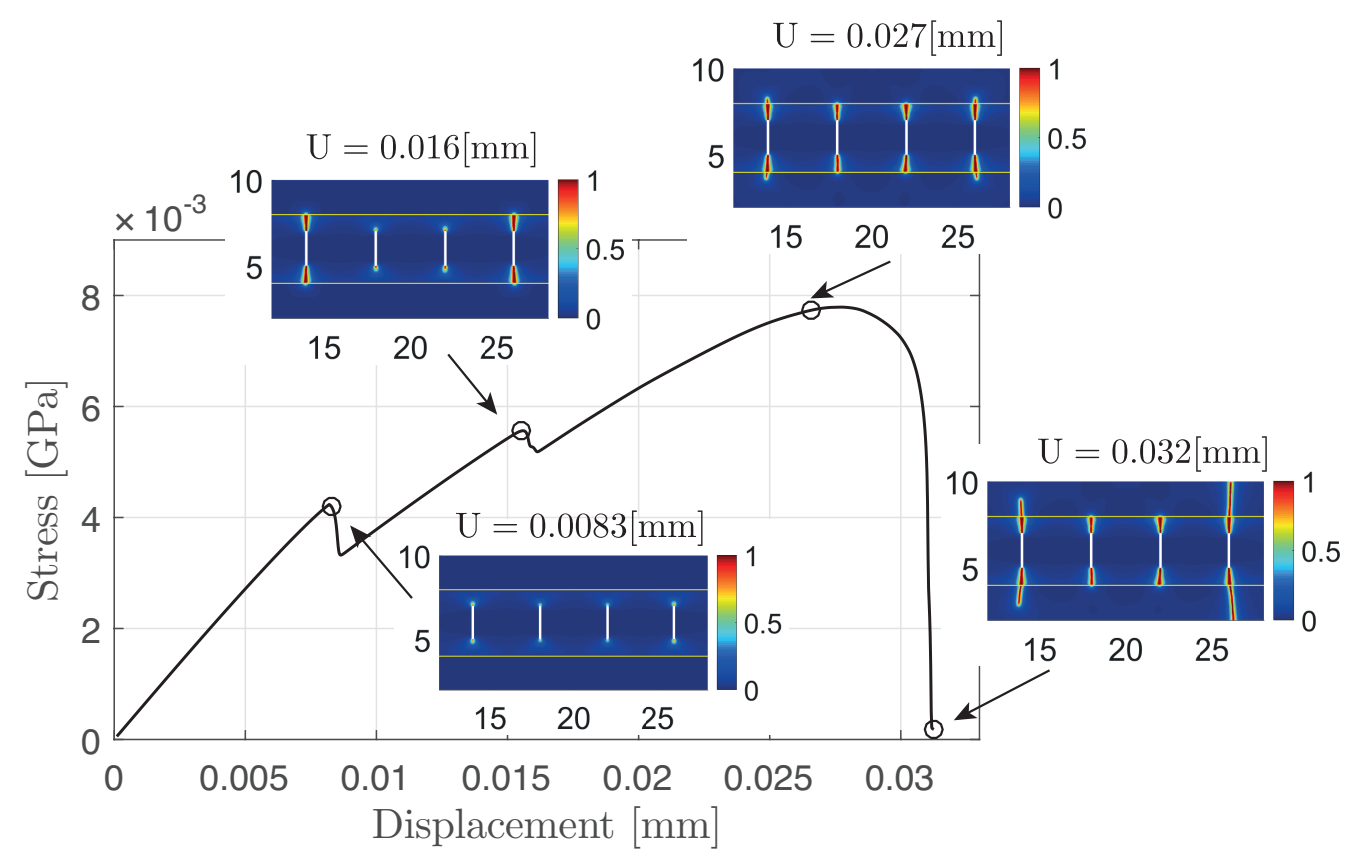

Fig. 18. Sharp transition - perfect interface case (C1): Crack path and stress - displacement curve, zoom in the interested region $(x=$ $[1228] ; y=[210])$

Next we analyze the results that are calculated by the cohesive interface (C2). The onset of two initial cracks (IC1) and (IC4) is observed as the previous case (C1) but at smaller loading capacity (see Fig. 19). So that after the prescribed displacement $U=0.0083 \mathrm{~mm}$, the structure has quickly lost its resistance. This phenomenon is due to the decohesion of the interfaces between central layer and top-bottom layers, which happened at the moment of crack reaching the interfaces $U=0.013 \mathrm{~mm}$. Therefore, it reduces the supporting among those layers and effectively loses the material resistance. The crack propagation of the outer cracks (IC1) and (IC4) significantly dominates over that of the inner ones. The inner cracks (IC2) and (IC3) initially grow but are then arrested within the center layer (i.e., no growth anymore), whereas severely growing is found for the outer cracks. They are even branching at the interfaces $U=0.022 \mathrm{~mm}$. However, an interesting point is captured, the interfacial decohesion oppositely avoids the crack penetration into the top and bottom layers (see Fig. 19 at $U=0.033 \mathrm{~mm}$ ). As a consequence, at this loading, structure behaves as three sub-structures separately, where the center layer has lose totally its resistance but the up and down layers is still intact. So that the structure still resist even at very high loading.

The simulation results for the last case, the regularized transition interface (C3), are depicted in Fig. 20. We capture here the similar phenomena with the case $(\mathrm{C} 1)$ for onset and reaching the interfaces of two initial cracks (IC1) and (IC4). Again the same behavior of cracks propagation for (IC2) at the prescribed displacement $U=0.0162$ $\mathrm{mm}$ is obtained. However, the smooth transition property of the interface in this case completely alters the behavior 


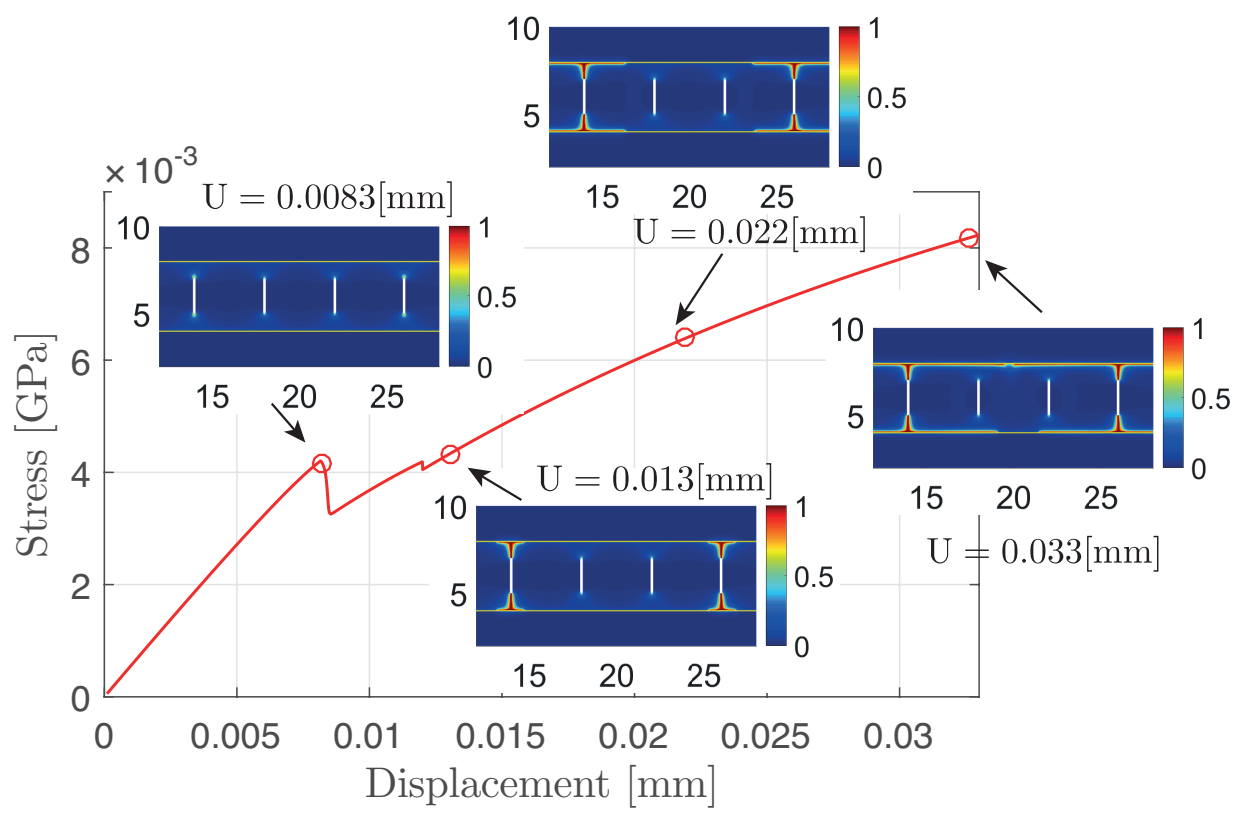

Fig. 19. Sharp transition - cohesive interface case (C2): Crack path and stress - displacement curve, zoom in the interested region $(x=$

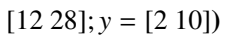

of structure for the next loading period. No crack branching for the (IC1) and (IC4) holds. Instead, the cracks penetrate into the top and bottom layers, leading to the loss of material resistance as can be observed from Fig. 20 at $U=0.0296 \mathrm{~mm}$. Similar to the case (C1), the damage of structure is mainly caused by the onset of the multiple cracks, not that by the delamination among layers.

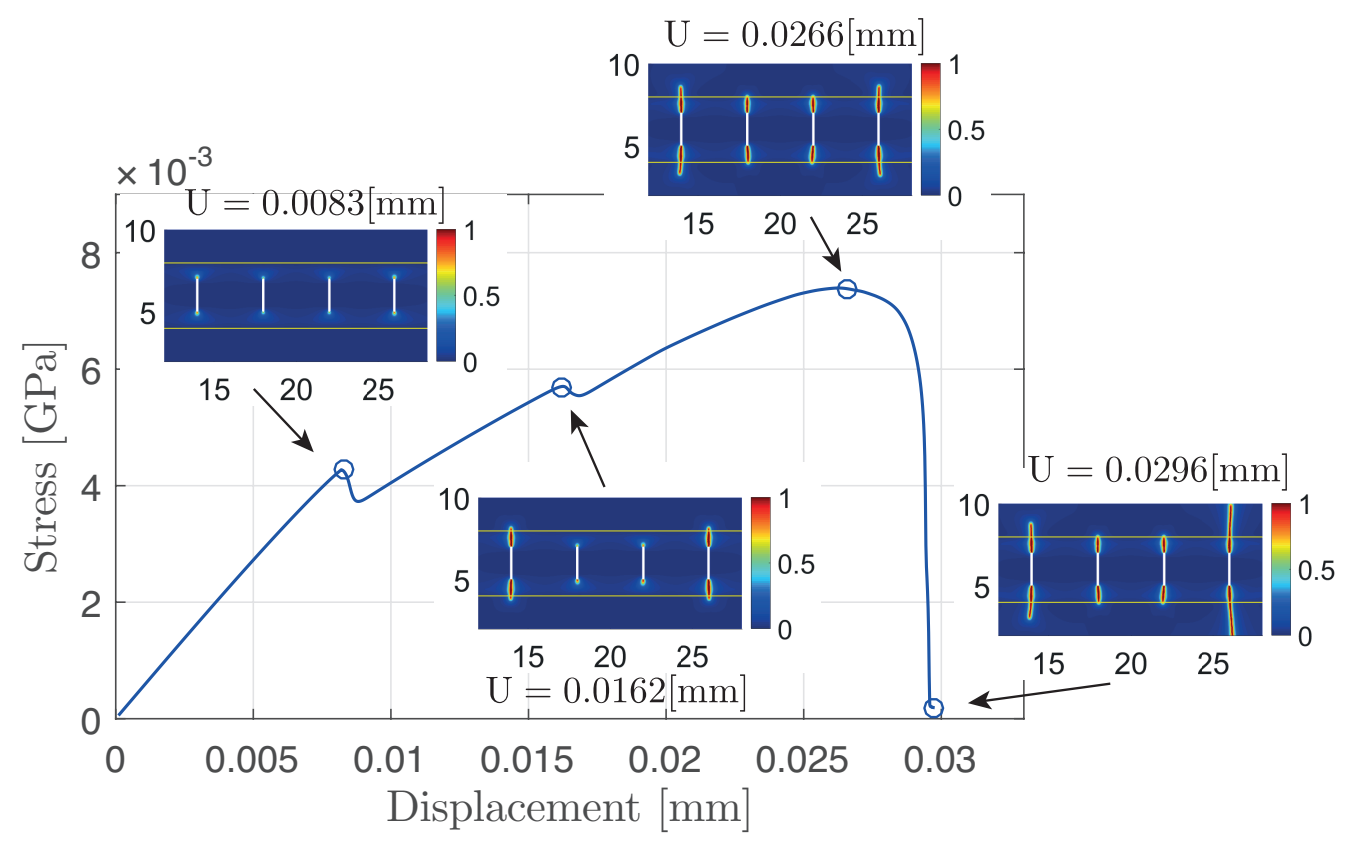

Fig. 20. Regularized transition - perfect interface case $(\mathbf{C 3})$ with $\ell_{\text {Trans }} / \ell=2$ : Crack path and stress - displacement curve, zoom in the interested region $(x=[1228] ; y=[210])$ 
To further interpretation of the results, a comparison of the structural stress-displacement curves during the entire loading history, and the final fracture state for all three situations is depicted in Fig. 21, gaining some interesting phenomena. It is important to highlight the difference on the damage mechanism among three types of considered interfaces. A switching of failure mode from bulk fracture to delamination along the interface $(\mathrm{C} 3) \rightarrow(\mathrm{C} 1) \rightarrow(\mathrm{C} 2)$ is obtained, providing the improvement of the structure resistance at different loading period. More specifically, the obtained results demonstrate that the interfacial nature (C3) exhibits best mechanical performance in loading phase 1 with $(U<0.020 \mathrm{~mm}),(\mathrm{C} 1)$ in loading phase 2 with $(U=0.020-0.028 \mathrm{~mm})$, and $\mathrm{C} 2$ in loading phase 3 with $(U>0.028 \mathrm{~mm})$. These phenomena may provide some helpful information for material manufacturing.

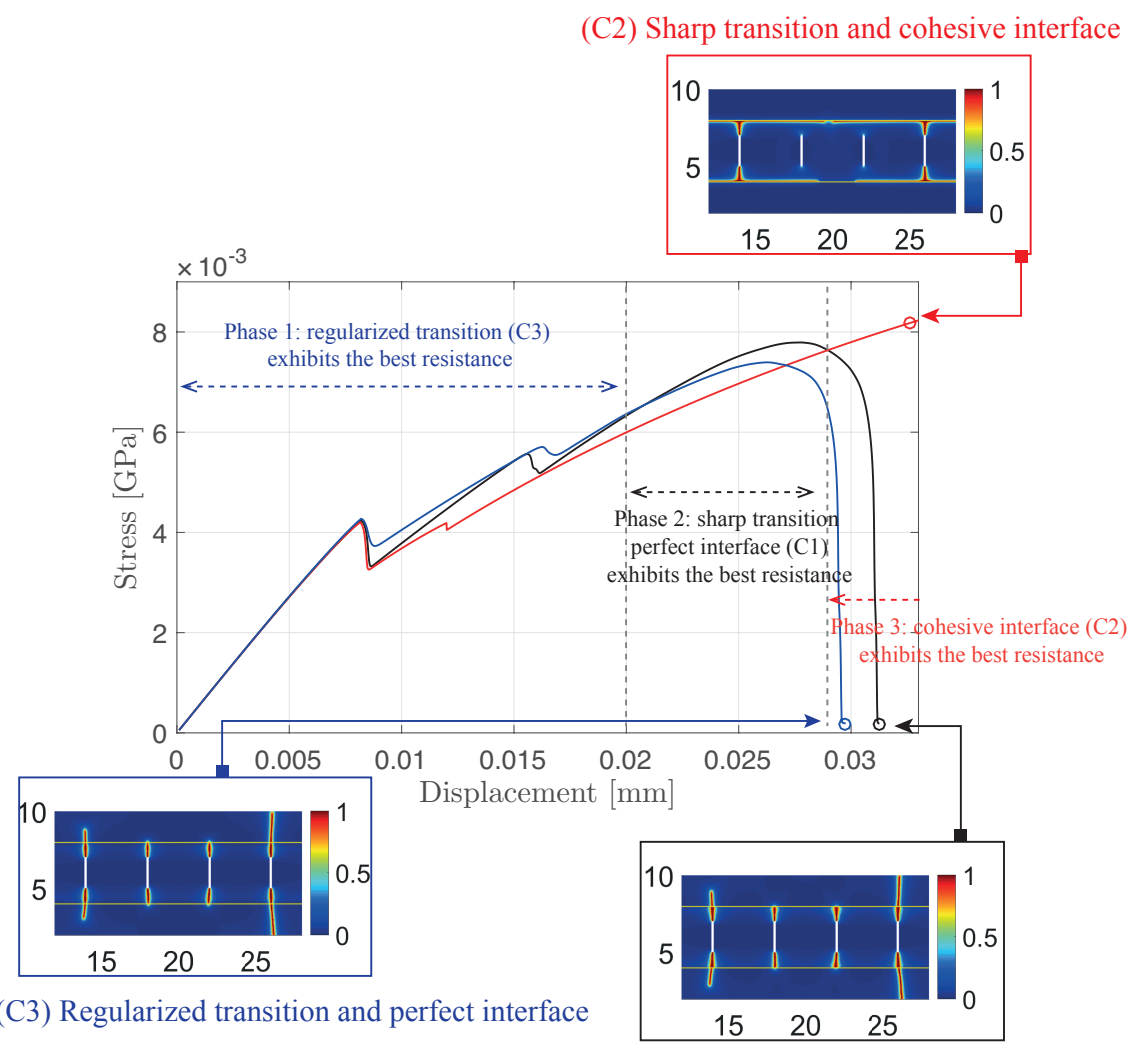

(C1) Sharp transition and perfect interface

Fig. 21. Comparison of crack paths and stress-displacement curves among (C1), (C2), and (C3), with $\ell_{\text {Trans }} / \ell=2$, zoom in the interested region $(x=[1228] ; y=[210])$

\subsection{Experimental validation: Crack growth in a multi-layered structure}

In the last example, we investigate the crack onset and propagation in more involved test and quantitatively compare the predicted crack paths with the experimental data provided by $[9,61]$. Our main goal here is to quantitatively evaluate whether the developed phase field approach is able to predict the evolution of crack in an more complex configuration.

Let us consider a multi-layered structure $\left(L \times B=44.5 \times 4.0 \mathrm{~mm}^{2}\right)$ as depicted in Fig. 22, consisting of 5 ATZ layers (made of $\mathrm{Al}_{2} \mathrm{O}_{3}$ with $5 \%$ tetragonal $\mathrm{ZrO}_{2}$ ) and $4 \mathrm{AMZ}$ layers (made of $\mathrm{Al}_{2} \mathrm{O}_{3}$ with $30 \%$ monoclinic $\mathrm{ZrO}_{2}$ ). A sharp notched sample with a length of $a=0.3 \mathrm{~mm}$ is considered. The structure under consideration is loaded in four-point bending, i.e., inner and outer spans: 20 and $40 \mathrm{~mm}$, respectively. The detail of the geometry and boundary conditions of the multi-layered structure can be found in Fig. 22.

The material parameters for this example listed in Table 4 are given by [61]. As discussed above, the length-scale parameter $\ell$ is a regularized parameter controlling the width of the smooth approximation of the crack. Nevertheless, 


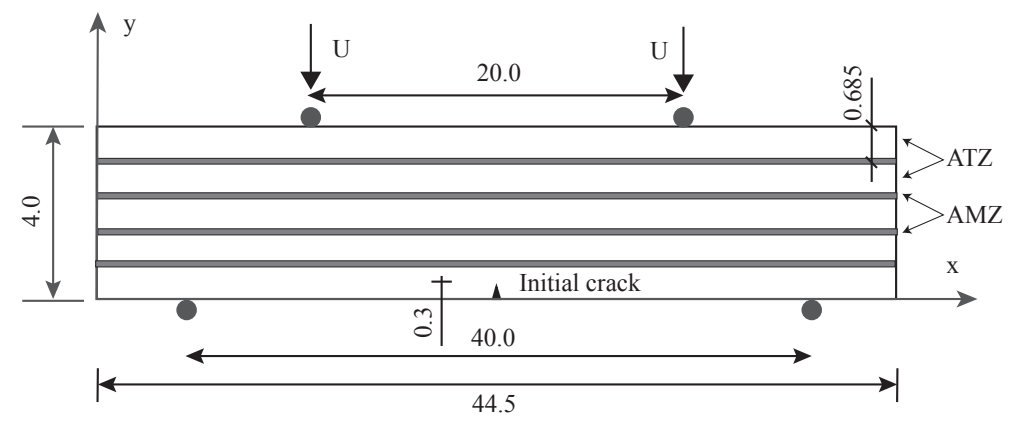

Fig. 22. Schematic of geometry and boundary conditions for a multi-layered structure according to the experimental set-up in [61] (unit in mm)

\begin{tabular}{|c|l|l|l|l|l|}
\hline Material & $E(\mathrm{GPa})$ & $v$ & $g_{c}(\mathrm{~N} / \mathrm{m})$ & $\sigma_{c}(\mathrm{MPa})$ & $\ell(\mathrm{mm})$ \\
\hline ATZ & 390 & 0.22 & 26 & 450 & 0.005 \\
AMZ & 280 & 0.22 & 21 & 110 & 0.005 \\
\hline
\end{tabular}

Table 4. Material parameters used for the multi-layered structure depicted in Fig. 23.

$\ell$ is also an internal parameter (related to the material properties) that influences the critical stress at which crack nucleation occurs. The detailed investigation of its effects on the fracture phenomena can be found in [55, 65]. The regularization parameter $\ell$ could be identified from the relation with material parameters, following $[49,5,56]$ :

$$
\ell=\frac{27 E g_{c}}{256 \sigma_{c}^{2}}
$$

Another constraint must be considered, the internal length $\ell$ should be taken as small as possible with respect to characteristic length of considered structure [55, 65]. In the case of ATZ material with $E=390 \mathrm{GPa}, g_{c}=26 \mathrm{~N} / \mathrm{m}$ and $\sigma_{c}=450 \mathrm{MPa}, \ell_{\mathrm{ATZ}} \approx 0.005 \mathrm{~mm}$ is thus obtained. This is small enough compared to the size of ATZ layer $(0.685$ $\mathrm{mm}$ ). However, for the AMZ material, the internal length is about $\approx 0.050 \mathrm{~mm}$. Clearly, this value is too large in comparison with the corresponding layer size $\approx 0.1450 \mathrm{~mm}$, and will not ensure the convergence to the brittle fracture theory of phase field model. In the heterogeneous configuration, the choice of different value $\ell$ for each region (each material) leading to the smeared crack, which is either thicker or thinner when propagating from one material to the other. This is a critical point in terms of fracture mechanics, especially in the multi-physics problems considering the effects of damaged zone. The fundamental of the physical meaning of the internal length in heterogeneous materials is challenging. Accurate procedures to characterize this parameter for the heterogeneous media are still an open question, and must be conducted.

In this work, we aim to validate qualitatively crack path with respect to the experimental observation. Hence, for the sake of simplicity, the internal length is chosen homogeneous $\ell=0.005 \mathrm{~mm}$ for whole domain. FE simulations with a refined mesh of triangular elements under plane strain assumption are conducted by using the (C1) interfacial modeling. Similarly, $h_{\max }=0.5 \mathrm{~mm}$ and $h_{\min }=0.0025 \mathrm{~mm}$ in the critical region to satisfy the condition $h_{\min } \leq \ell / 2$ are taken. The monotonic compressive displacement $\Delta U=-3 \times 10^{-4} \mathrm{~mm}$ is prescribed as long as the phase field $d<0.8$ in all elements, and $\Delta U=-5 \times 10^{-5}$ as soon as $d \geq 0.8$ in one integration point.

Fig. 23 shows the computed numerical prediction of crack propagation at several loading steps. It can be observed from the figures that, under such loading and boundary conditions, the crack starts propagating from the designed notch, reaches the interface and then branches at the second layer of AMZ. The crack in one wing subsequently penetrates through the next ATZ layers. Again, a small crack branching was observed at second AMZ layer before across the third ATMZ layer. The phenomena of crack delamination and deflection at the interface can be explained as the same principle discussed in subsection 5.3. 


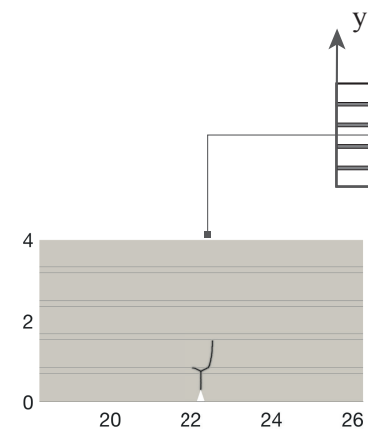

(a)

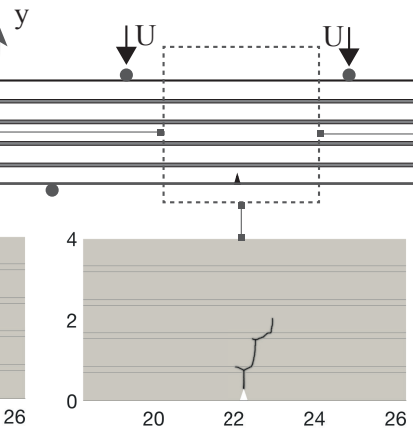

(b)

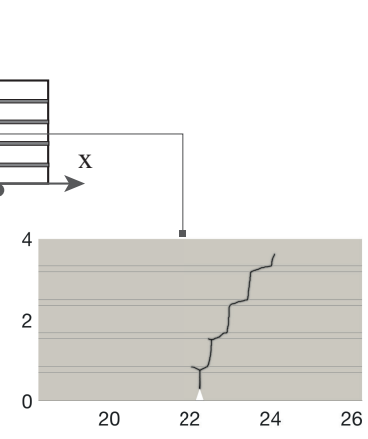

(c)

Fig. 23. Crack propagation in a multi-layered structure at several loading steps obtained by the present phase field model.

In Fig. 24, we show a qualitative comparison of crack path profile between the numerical simulation and the experimental result ([61]). Clearly, the developed numerical prediction based on the phase filed model captures well the "step-like" fracture of a layered ceramic structure. The crack path profile agrees well with the experimental observation ([61]), which further confirms the applicability and accuracy of the proposed model in studying complex fracture phenomena in layered heterogeneous structures.
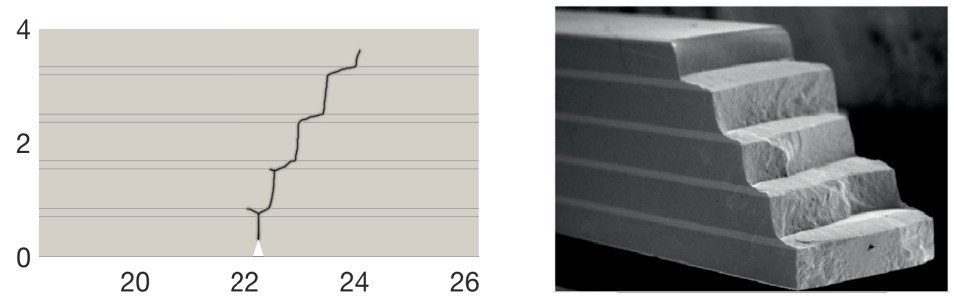

Fig. 24. Comparison of crack morphologies between numerical prediction (left) and experiment (right) ([61]). The step-like fracture obtained by the present model agrees well with experimental observation.

\section{Conclusions and outlook}

This contribution has outlined the development of a variational phase field model for simulation of crack-interface interactions in 2-D and 3-D layered structures, exploring the effects of the interface on fracture behavior. In general, this variational phase field model offers self-consistent descriptions of fracture, without the need for ad hoc fracture criteria, conventionally capturing the onset of cracks, especially multiple cracks and 3-D case. On the theoretical aspect, we address the characteristics of the interfaces, highlighting the constitutive relations on the layered material interfaces. The regularized interfacial transition zone in terms of the regularized phase field model is introduced. The phase field models for both perfectly bonded and cohesive interfaces with sharp and regularized transitions are described. On the computational and numerical aspects, we analyze the role of the regularized interfacial transition zone and its performance fulfilling both 2-D and 3-D layered systems. Different fracture phenomena depending on mechanical characterization of interface are investigated and observed. The crack growth, merging and branching are modeled in the very efficient way. The obtained results reproduce very well the analytical prediction given by $[35,36]$. Specially, the numerical simulation captures a complex phenomenon of crack crossing the interface between two layers, that is a difficult and challenging task to the previous models.

This work provides a feasible phase field approach based on the regularized interfacial transition to model the interface regions, where the smooth transition may exist. It is to underline that the regularized transition model behaves similarly to the Heaviside function, but in the smeared phase field context instead. It means that the sharp 
description model can be recovered by the new definition in a straightforward manner, which is by simply setting the defined regularized transition length to be null. This description to the interface modeling is, we believe, more flexible and effective compared with the conventional approaches. We have analyzed the effects of interfacial transition zone on the crack onset of simple and complex situation, wherein layered structures evolve single or multiple cracks. Some major conclusions drawn from the study can be summarized as follows:

- The developed phase field approach has proved to be effective in modeling crack-interface interactions in 2-D and 3-D structures, reflecting the more flexibility of the proposed regularized interfacial transition approach.

- The material mismatch ratio has a strong influence on the fracture behavior at the interface in layered structures, showing a substantial dependence of crack penetration and branching on the material mismatch.

- The characteristics of fracture at the interface in layered structures generally depend on the defined interface modelings. In this study, the developed models such as (C1), (C2) and (C3) greatly affect the crack paths (e.g., penetration, branching) at the interfaces. In addition, our studies also indicate a strong influence of transition zone on crack paths and damage mechanism, and especially on the ability of crack crossing the interface.

- Damage mechanisms of multiple cracked specimens are very complex. It is again found the significant influence of the three considered interface models on the evolution of cracks in multiple layered structures. The observed results on engineering applications to multiple cracks with fracture spacing in three-layered structure and experimental validation of a multi-layered structure one more time confirm the important role of the interfacial behavior on the structure resistance. Different kinds of interface can reinforce the material at different loaded period. Note that these properties evidently play a key point on the design of functionally graded materials.

With the confidence established by the favorable investigations to 2-D and 3-D models, and to simple and complex crack structures, it is believed that the variational phase field models for fracture in layered heterogeneous structures can stand as a robust and effective tool in understanding many of the more complex fracture behavior that occur in a wide range of advanced engineering materials such as functionally graded media and laminated structures.

The current work is devoted to the analysis of mechanical fracture characteristics of interfacial transition zone problems under quasi-static loading condition. However, the effects of dynamic loads on fracture in layered heterogeneous structures in terms of phase field model are important and interesting. Extension of the present models to take into account dynamic loading condition effects is in general quite possible, but such a dynamic fracture model would be very challenging in both physical fundamentals and numerical implementation. This interesting work is out of the scope of the current study but has already been scheduled for our future studies.

\section{Acknowledgments}

The support this work enjoys from SeRaMCo project (Secondary Raw Materials for Concrete Precast Products), through the transnational cooperation projects in North-West Europe (Interreg NWE), NWE-320 is gratefully acknowledged. TQB gratefully acknowledges the internal grant supported by Tokyo Institute of Technology, Japan. The authors gratefully thank all the anonymous reviewers for their constructive suggestions and valuable comments to improve the quality of the manuscript.

\section{Appendix A. Fundamental of thermodynamics and evolution of phase field}

In this section, we present formulation for a crack phase field evolution law that can guarantee the irreversibility of the process. Assuming isothermal process and without the external mircoforces, the Clausius-Duhem inequality can be written by introducing a specific dissipation function per unit volume $\mathcal{D}$ as follows:

$$
\mathcal{D}=\sigma: \dot{\varepsilon}-\dot{\psi} \geq 0
$$


By using the definition of the energy density $\psi$ given in Eq. (7), the local dissipation (A.1) can be rewritten as

$$
\mathcal{D}=\left(\sigma-\frac{\partial \psi}{\partial \boldsymbol{\varepsilon}}\right): \dot{\boldsymbol{\varepsilon}}+\mathcal{A}_{d} \dot{d} \geq 0
$$

where $\mathcal{A}_{d}=-\frac{\delta \psi}{\delta d}=-\frac{\partial \psi}{\partial d}+\nabla \cdot\left(\frac{\partial \psi}{\partial \nabla d}\right)$ is the variational derivative of $\psi$ with respect to the phase field $d$.

With the assumption that the elastic strain rate $\dot{\varepsilon}$ can have arbitrarily prescribed values and is independent of the rate of the order parameter, and applying the Coleman's exploitation, Eq. (A.2) yields the constitutive relation for the stress $\sigma$ as

$$
\sigma=\partial_{\varepsilon} \psi
$$

A reduced form of the Clausius-Duhem inequality can be now re-written by

$$
\mathcal{A}_{d} \dot{d} \geq 0 .
$$

At this stage, a threshold function $F\left(\mathcal{A}_{d}\right)$ such that

$$
F\left(\mathcal{A}_{d}\right) \leq 0
$$

is introduced. Assuming the principle of maximum dissipation requires the dissipation $\mathcal{A}_{d} \dot{d}$ to be maximum under the above constraint Eq. (A.5). Using the method of Lagrange multipliers, which defines the following Lagrangian

$$
\mathcal{L}=-\mathcal{A}_{d} \dot{d}+\lambda F\left(\mathcal{A}_{d}\right)
$$

yields the Kuhn-Tucker equations

$$
\frac{\partial \mathcal{L}}{\partial \mathcal{A}_{d}}=0, \lambda \geq 0, \quad F \leq 0, \lambda F=0 .
$$

The first equality in Eq. (A.7) gives

$$
\dot{d}=\lambda \frac{\partial F\left(\mathcal{A}_{d}\right)}{\partial \mathcal{A}_{d}} .
$$

Without loss of generality, the threshold function $F\left(\mathcal{A}_{d}\right)$ is assumed in the form $F\left(\mathcal{A}_{d}\right)=\mathcal{A}_{d}$. From Eq. (A.8) and using the second inequality in Eq. (A.7), we reach

$$
\dot{d}=\lambda \geq 0 .
$$

For $\dot{d}>0$, and from Eqs. (A.4), (A.9) and the third equality in Eq. (A.7), which give $F=0$, implying

$$
F=-\frac{\delta \psi}{\delta d}=-\frac{\partial \psi_{u}}{\partial d}-g_{c} \delta \gamma(d, \nabla d)=0
$$

with (see e.g., [41])

$$
\delta \gamma(d, \nabla d)=\frac{d}{\ell}-\ell \Delta d
$$

From Eqs. (8) and (A.10), one obtains

$$
2(1-d) \psi_{u}^{+}-g_{c} \delta \gamma(d, \nabla d)=0 .
$$

The local crack driving force $\mathcal{H}(t)$ is introduced to describe a dependence on history [41] and to make possible loading-unloading

$$
\mathcal{H}(\mathbf{x}, t)=\max _{\tau \in[0, t]}\left\{\frac{\ell}{g_{c}} \psi^{+}(\mathbf{x}, \tau)\right\},
$$


Basically, $\mathcal{H}(\mathbf{x}, t)$ contains the maximum reference energy, or a measure for the maximum tensile strain obtained in the loading history, which provides a very transparent representation of the balance equation that governs the diffusive crack topology. For more detail, curious readers should refer to references, e.g., [41, 50]. From (A.11), (A.12), and (A.13).

The evolution law for phase field is finally expressed as

$$
2(1-d) \mathcal{H}-\left(d-\ell^{2} \Delta d\right)=0 \text { in } \Omega,
$$

belong with a homogeneous Neumann condition $\nabla d(\mathbf{x}) \cdot \mathbf{n}=0$ on $\partial \Omega$.

The irreversible character of the crack evolution law described in Eq. (A.14) can be verified by following. As $\mathcal{H}(\mathbf{x}, t) \geq 0$, the Eq. (A.12) implies

$$
\delta \gamma(d, \nabla d) \geq 0 .
$$

We can thus check that due to Eqs. (A.9), (A.15) the variation of crack length

$$
\dot{\Gamma}_{l}=\int_{\Omega} \delta \gamma(d, \nabla d) \dot{d} \mathrm{~d} \Omega \geq 0
$$

satisfying the criteria of irreversible evolution of cracks.

\section{References}

[1] Aboushelib, M., de N. Jager, Kleverlaan, C., Feilzer, A., 2007. Effect of loading method on the fracture mechanics of two layered all-ceramic restorative systems. Dental Materials 23 (8), 952-959.

[2] Ambrosio, L., Tortorelli, V., 1990. Approximation of functional depending on jumps by elliptic functional via $\Gamma$-convergence. Communications on Pure and Applied Mathematics 43 (8), 999-1036.

[3] Ambrosio, L., Tortorelli, V., 1990. Approximation of functionals depending on jumps by elliptic functionals via $\Gamma$-convergence. Communications on Pure and Applied Mathematics 43, 999-1036.

[4] Ambrosio, L., Tortorelli, V., 1992. On the approximation of free discontinuity problems. Boll. U.M.I. 6-B, 105-123.

[5] Amor, H., Marigo, J., Maurini, C., 2009. Regularized formulation of the variational brittle fracture with unilateral contact: Numerical experiments. Journal of the Mechanics and Physics of Solids 57 (8), 1209-1229.

[6] Bai, T., Pollard, D., Gao, H., 2000. Explanation for fracture spacing in layered materials. Nature 403 (6771), 753-756.

[7] Bao, G., Wang, L., 1995. Multiple cracking in functionally graded ceramic/metal coatings. International Journal of Solids and Structures 32 (19), 2853-2871.

[8] Barenblatt, G., 1959. The formation of equilibrium cracks during brittle fracture. General ideas and hypotheses. axially-symmetric cracks. Journal of Applied Mathematics and Mechanics 23 (3), 622-636.

[9] Bermejo, R., Šestáková, L., Grünbichler, H., Lube, T., Supancic, P., Danzer, R., 2011. Fracture mechanisms of structural and functional multilayer ceramic structures. In: Key Engineering Materials. Vol. 465. Trans Tech Publ, pp. 41-46.

[10] Bhattacharya, S., Singh, I., Mishra, B., Bui, T. Q., 2013. Fatigue crack growth simulations of interfacial cracks in bi-layered fgms using xfem. Computational Mechanics 52, 799-814.

[11] Borden, M., Hughes, T., Landis, C., Verhoosel, C., 2014. A higher-order phase-field model for brittle fracture: Formulation and analysis within the isogeometric analysis framework. Computer Methods in Applied Mechanics and Engineering 273, $100-118$.

[12] Bourdin, B., Francfort, G., Marigo, J., 2000. Numerical experiments in revisited brittle fracture. Journal of the Mechanics and Physics of Solids 48 (4), 797-826.

[13] Bourdin, B., Larsen, C., Richardson, C., 2011. A time-discrete model for dynamic fracture based on crack regularization. International Journal of Fracture 168, 133-143.

[14] Bourdin, B., Marigo, J.-J., Maurini, C., Sicsic, P., 2014. Morphogenesis and propagation of complex cracks induced by thermal shocks. Physical review letters 112 (1), 014301.

[15] Braides, A., 1998. Approximation of free-discontinuity problems. No. 1694. Springer Science \& Business Media.

[16] Braides, D., 1998. Approximation of Free Discontinuity Problems. Springer Verlag: Berlin.

[17] Braides, D., 2002. Г-Convergence for Beginners. Oxford University Press: New York.

[18] Cuvilliez, S., Feyel, F., Lorentz, E., Michel-Ponnelle, S., 2012. A finite element approach coupling a continuous gradient damage model and a cohesive zone model within the framework of quasi-brittle failure. Computer methods in applied mechanics and engineering 237, $244-259$.

[19] de Borst, R., Verhoosel, C., 2016. A discussion on gradient damage and phase-field models for brittle fracture. In: Advanced Methods of Continuum Mechanics for Materials and Structures. Springer, pp. 263-277.

[20] den Bosch, M. V., Schreurs, P., Geers, M., 2006. An improved description of the exponential xu and needleman cohesive zone law for mixed-mode decohesion. Engineering Fracture Mechanics 73 (9), 1220-1234. 
[21] Doan, D. H., Bui, T. Q., Do, T. V., Nguyen, D. D., 2017. A rate-dependent hybrid phase field model for dynamic crack propagation. Journal of Applied Physics 122, 115102.

[22] Doan, D. H., Bui, T. Q., Nguyen, D., Fushinobu, K., 2016. Hybrid phase field simulation of dynamic crack propagation in functionally graded glass-filled epoxy. Composites Part B: Engineering 99, 266-276.

[23] Dugdale, D., 1960. Yielding of steel sheets containing slits. Journal of the Mechanics and Physics of Solids 8, 100-104.

[24] Francfort, G., Marigo, J., 1998. Revisiting brittle fracture as an energy minimization problem. Journal of the Mechanics and Physics of Solids 46 (8), 1319-1342.

[25] Freddi, F., Iurlano, F., 2017. Numerical insight of a variational smeared approach to cohesive fracture. Journal of the Mechanics and Physics of Solids 98, 156-171.

[26] Grujicic, M., Zhao, H., 1998. Optimization of 316 stainless steel/alumina functionally graded material for reduction of damage induced by thermal residual stresses. Materials Science and Engineering: A 252 (1), 117-132.

[27] Hakim, V., Karma, A., 2009. Laws of crack motion and phase-field models of fracture. Journal of the Mechanics and Physics of Solids 57 (2), 342-368.

[28] He, M., Heredia, F., Wissuchek, D., Shaw, M., Evans, A., 1993. The mechanics of crack growth in layered materials. Acta metallurgica et materialia 41 (4), 1223-1228.

[29] Jin, Z.-H., Batra, R., 1996. Some basic fracture mechanics concepts in functionally graded materials. Journal of the Mechanics and Physics of Solids 44 (8), 1221-1235.

[30] Jin, Z.-H., Paulino, G., Dodds, R., 2003. Cohesive fracture modeling of elastic-plastic crack growth in functionally graded materials. Engineering Fracture Mechanics 70 (14), 1885-1912.

[31] Karma, A., Kessler, D., Levine, H., 2001. Phase-field model of mode III dynamic fracture. Physical Review Letters 87 (4), 45501.

[32] Khaderi, S., Murali, P., Ahluwalia, R., 2014. Failure and toughness of bio-inspired composites: Insights from phase field modelling. Computational Materials Science 95, 1-7.

[33] Khandelwal, A., Kumar, A., Ahluwalia, R., Murali, P., 2017. Crack propagation in staggered structures of biological and biomimetic composites. Computational Materials Science 126, 238-243.

[34] Kuhn, C., Müller, R., 2010. A continuum phase field model for fracture. Engineering Fracture Mechanics 77 (18), $3625-3634$.

[35] Leguillon, D., Martin, E., 2013. The strengthening effect caused by an elastic contrast part i: the bimaterial case. International journal of fracture $179(1-2), 157-167$.

[36] Leguillon, D., Martin, E., 2013. The strengthening effect caused by an elastic contrast part ii: stratification by a thin stiff layer. International journal of fracture 179 (1-2), 159-178.

[37] Levitas, I. V., Javanbakht, M., 2011. Surface-induced phase transformations: multiple scale and mechanics effects and morphological transitions. Physical Review Letters 107, 175701.

[38] Levitas, I. V., Samani, K., 2014. Melting and solidification of nanoparticles: Scale effects, thermally activated surface nucleation, and bistable states. Physical Review B 89, 075427.

[39] Liu, X., Xiao, Q., Karihaloo, B., 2004. Xfem for direct evaluation of mixed mode SIFs in homogeneous and bi-materials. International Journal for Numerical Methods in Engineering 59 (8), 1103-1118.

[40] Maso, G. D., 1993. An Introduction to $\Gamma$-Convergence. Birkhäuser Verlag: Boston.

[41] Miehe, C., Hofacker, M., Welschinger, F., 2010. A phasefield model for rate-independent crack propagation: Robust algorithmic implementation based on operator splits. Computer Methods in Applied Mechanics and Engineering 199, 2765-2778.

[42] Miehe, C., Welschinger, F., Hofacker, M., 2010. Thermodynamically consistent phase-field models of fracture: Variational principles and multi-field fe implementations. International Journal for Numerical Methods in Engineering 83 (10), 1273-1311.

[43] Moës, N., Dolbow, J., Belytschko, T., 1999. A finite element method for crack growth without remeshing. International Journal for Numerical Methods in Engineering. 46(1), 131-156.

[44] Mumford, D., Shah, J., 1989. Optimal approximations by piecewise smooth functions and associated variational problems. Communications on Pure and Applied Mathematics. 42, 577-685.

[45] Murali, P., Bhandakkar, T., Cheah, W., Jhon, M., Gao, H., Ahluwalia, R., 2011. Role of modulus mismatch on crack propagation and toughness enhancement in bioinspired composites. Physical Review E 84 (1), 015102.

[46] Needleman, A., 1987. A continuum model for void nucleation by inclusion debonding. Journal of applied mechanics 54 (3), 525-531.

[47] Nguyen, T.-T., Réthoré, J., Yvonnet, J., Baietto, M.-C., 2017. Multi-phase-field modeling of anisotropic crack propagation for polycrystalline materials. Computational Mechanics 60, 289-314.

[48] Nguyen, T.-T., Yvonnet, J., Bornert, M., Chateau, C., 2016. Initiation and propagation of complex 3D networks of cracks in heterogeneous quasi-brittle materials: Direct comparison between in situ testing-microct experiments and phase field simulations. Journal of the Mechanics and Physics of Solids 95, $320-350$.

[49] Nguyen, T.-T., Yvonnet, J., Bornert, M., Chateau, C., Sab, K., Romani, R., Roy, R. L., 2016. On the choice of parameters in the phase field method for simulating crack initiation with experimental validation. International Journal of Fracture 197 (2), 213-226.

[50] Nguyen, T.-T., Yvonnet, J., Zhu, Q.-Z., Bornert, M., Chateau, C., 2015. A phase field method to simulate crack nucleation and propagation in strongly heterogeneous materials from direct imaging of their microstructure. Engineering Fracture Mechanics 139, 18-39.

[51] Nguyen, T.-T., Yvonnet, J., Zhu, Q.-Z., Bornert, M., Chateau, C., 2016. A phase-field method for computational modeling of interfacial damage interacting with crack propagation in realistic microstructures obtained by microtomography. Computer Methods in Applied Mechanics and Engineering 312, $567-595$.

[52] Paggi, M., Reinoso, J., 2017. Revisiting the problem of a crack impinging on an interface: a modeling framework for the interaction between the phase field approach for brittle fracture and the interface cohesive zone model. Computer Methods in Applied Mechanics and Engineering 
$321,145-172$.

[53] Paulino, G., Jr, R. D., 2002. Finite element investigation of quasi-static crack growth in functionally graded materials using a novel cohesive zone fracture model. Urbana 51, 61801.

[54] Pham, K., Amor, H., Marigo, J.-J., Maurini, C., 2011. Gradient damage models and their use to approximate brittle fracture. International Journal of Damage Mechanics 20 (4), 618-652.

[55] Pham, K., Marigo, J.-J., Maurini, C., 2011. The issues of the uniqueness and the stability of the homogeneous response in uniaxial tests with gradient damage models. Journal of the Mechanics and Physics of Solids 59 (6), 1163-1190.

[56] Pham, K., Ravi-Chandar, K., Landis, C., 2017. Experimental validation of a phase-field model for fracture. International Journal of Fracture 205 (1), 83-101.

[57] Pinchin, D., Tabor, D., 1978. Interfacial contact pressure and frictional stress transfer in steel fibre cement. Testing and Test Methods of Fibre Cement Composites, 337-344.

[58] Rahimzadeh, T., Arruda, E., Thouless, D., 2015. Design of armor for protection against blast and impact. Journal of the Mechanics and Physics of Solids 85, 98-111.

[59] Ravichandran, K., 1995. Thermal residual stresses in a functionally graded material system. Materials Science and Engineering: A 201 (1-2), 269-276.

[60] Schöpfer, M., Arslan, A., Walsh, J., Childs, C., 2011. Reconciliation of contrasting theories for fracture spacing in layered rocks. Journal of Structural Geology 33 (4), 551-565.

[61] Ševeček, O., Bermejo, R., Kotoul, M., 2013. Prediction of the crack bifurcation in layered ceramics with high residual stresses. Engineering Fracture Mechanics 108, 120-138.

[62] Shim, D.-J., Paulino, G., Dodds, R., 2006. J resistance behavior in functionally graded materials using cohesive zone and modified boundary layer models. International Journal of Fracture 139 (1), 91-117.

[63] Sukumar, N., Huang, Z., Prévost, J.-H., Suo, Z., 2004. Partition of unity enrichment for bimaterial interface cracks. International journal for numerical methods in engineering 59 (8), 1075-1102.

[64] Tang, C., Liang, Z., Zhang, Y., Chang, X., Tao, X., Wang, D., Zhang, J., Liu, J., Zhu, W., Elsworth, D., 2008. Fracture spacing in layered materials: a new explanation based on two-dimensional failure process modeling. American Journal of Science 308 (1), 49-72.

[65] Tanné, E., Li, T., Bourdin, B., Marigo, J.-J., Maurini, C., 2017. Crack nucleation in variational phase-field models of brittle fracture.

[66] Verhoosel, C., Borst, R. D., 2013. A phase-field model for cohesive fracture. International Journal for numerical methods in Engineering $96(1), 43-62$.

[67] Vignollet, J., May, S., Borst, R. D., Verhoosel, C., 2014. Phase-field models for brittle and cohesive fracture. Meccanica 49 (11), $2587-2601$.

[68] Wells, J., Beaumont, P., 1985. Debonding and pull-out processes in fibrous composites. Journal of Materials Science 20 (4), $1275-1284$.

[69] Wu, J. Y., 2017. A unified phase-field theory for the mechanics of damage and quasi-brittle failure. Journal of the Mechanics and Physics of Solids 103, 72-99.

[70] Xu, X.-P., Needleman, A., 1993. Void nucleation by inclusion debonding in a crystal matrix. Modelling and Simulation in Materials Science and Engineering 1 (2), 111.

[71] Yang, Q., Cox, B., 2005. Cohesive models for damage evolution in laminated composites. International Journal of Fracture 133 (2), $107-137$.

[72] Yu, T., Bui, T. Q., Liu, P., Zhang, C., Hirose, S., 2015. Interfacial dynamic impermeable cracks analysis in dissimilar piezoelectric materials under coupled electromechanical loading with the extended finite element method. International Journal of Solids and Structures 67-68, $205-218$.

[73] Zi, G., Song, J., Budyn, E., Lee, S., Belytschko, T., 2004. A method for growing multiple cracks without remeshing and its application to fatigue crack growth. Modelling and Simulation in Materials Science and Engineering 12 (5), 901. 